\title{
Dynamics of flame extinction in narrow channels with cold walls: heat loss versus acceleration
}

Claude M. Dion, ${ }^{1}$ Damir M. Valiev, ${ }^{2,3,4, a)}$ V'yacheslav Akkerman, ${ }^{5}$ Berk Demirgok, ${ }^{5}$ Orlando $\mathrm{J}$. Ugarte, ${ }^{5}$

Lars-Erik Eriksson, ${ }^{6}$ and Vitaly Bychkov $\left.{ }^{1, b}\right)$

1) Department of Physics, Umeå University, SE-901 87 Umeå, Sweden

${ }^{2)}$ Department of Applied Physics and Electronics, Umeå University, SE-90187 Umeå,

Sweden

${ }^{3)}$ Center for Combustion Energy, Department of Energy and Power Engineering, Tsinghua University,

Beijing 100084, China

${ }^{4)}$ Key Laboratory for Thermal Science and Power Engineering of the Ministry of Education, Tsinghua University,

Beijing 100084, China

${ }^{5)}$ Center for Innovation in Gas Research and Utilization (CIGRU), Department of Mechanical and

Aerospace Engineering, West Virginia University, 1306 Evansdale Drive, Morgantown, WV 26506,

USA

${ }^{6)}$ Department of Applied Mechanics, Chalmers University of Technology, 41296 Gothenburg,

Sweden

Propagation of a premixed flame from a closed to an open end in micro-channels with smooth non-slip isothermal walls is considered in the context of flame extinction dynamics. Powerful exponential flame acceleration in micro-channels with adiabatic walls has been demonstrated at the initial quasi-isobaric stage of the process [V. Bychkov et al., Physical Review E 72, 046307 (2005)]. In contrast to the previous studies, here we investigate flame propagation in channels with isothermal walls. The problem is solved by means of high-fidelity laminar numerical simulations of the complete set of the Navier-Stokes combustion equations. For most of the problem parameter sets chosen, we obtain initial flame acceleration after ignition at the closed channel end. This acceleration resembles qualitatively the adiabatic case, but develops noticeably slower, in an approximately linear regime instead of the exponential one and persists only for a limited time interval. Subsequently, heat loss to the walls reduces the temperature and hence the volume of the burnt gas behind the flame front, which produces a reverse flow in the direction of the closed channel end. When the amount of the burnt gas becomes sufficiently large, the reverse flow stops the acceleration process and drives the flame backwards with modifications of the flame front shape from convex to concave. Eventually, the flame extinguishes. Qualitatively, the process obtained reproduces a possible combustion failure during deflagration-to-detonation transition observed in previous experiments. We investigate the key characteristics of initial flame acceleration such as the acceleration rate and the maximum speed of the flame tip.

Keywords: flame acceleration, isothermal wall, cold wall

\section{INTRODUCTION}

Spontaneous acceleration of an initially slow deflagration front is of great interest from both practical and fundamental viewpoints ${ }^{1,2}$. Prevention of flame acceleration (FA) is critically important in the context of fire safety and explosion mitigation, in particular, in application to flame arrestors ${ }^{3-5}$ and in the prevention of mining accidents ${ }^{6,7}$. At the same time, promotion of FA and efficient use of the deflagration-to-detonation transition (DDT) is an important problem for the development of novel combustion devices, such as pulsedetonation engines ${ }^{8,9}$. The key element of DDT is spontaneous FA, starting with a slow laminar flame and attaining supersonic propagation in the laboratory reference frame by the end of the process ${ }^{10,11}$. A typical geometry in experiments and energy-production devices employing FA corresponds to a relatively long channel,

a)Corresponding author; dvaliev@tsinghua.edu.cn b) Deceased with the flame propagating from the closed channel end to the open one ${ }^{8}$. Experimental activity on the subject is extremely high covering scales from micro-channels to huge pipes, i.e., from less than $1 \mathrm{~mm}$ to more than $1 \mathrm{~m}$ in diameter ${ }^{1,8,12-16}$. It is noted that using low-energy ignition to initiate the DDT is favored as an energetically beneficial way of attaining detonation.

In the present work we consider channels with smooth non-slip walls. A mechanism of FA in channels with non-slip at the walls has been qualitatively described by Shelkin ${ }^{17,18}$, according to which, a flame front in channels with smooth walls accelerates because of the thermal expansion of the burning gas and the non-slip boundary conditions at the walls. Expansion of the burning gas produces a flow of the fuel mixture, which becomes nonuniform due to the non-slip boundary conditions. The non-uniform flow makes the flame front curved, which increases the burning rate and creates a positive feedback between the flame and the flow, hence leading to FA. Although the qualitative idea of such acceleration was suggested by Shelkin already in the 1940 's ${ }^{17,18}$, the quantitative theory of the process has been developed 
by Bychkov et al. ${ }^{19}$ and supported by extensive numerical simulations ${ }^{19-21}$. Among other results, this theory ${ }^{19}$ predicted powerful exponential FA in micro-channels (at least at the initial, almost isobaric stage of the process), and a decrease of the scaled acceleration rate with the channel width characterized by the Reynolds number associated with flame propagation, $\operatorname{Re} \equiv S_{L} R / \nu$, where $S_{L}$ is the laminar flame speed, $R$ is the channel half-width (radius), and $\nu$ is the kinematic viscosity.

The theory and modelling ${ }^{19,20}$ involved inevitable simplifications and limitations, such as adiabatic and isobaric conditions, and required experimental confirmation. Novel experiments of this type have been designed and performed by $\mathrm{Wu}$ et al. ${ }^{13,14}$, and supported the conceptual possibility of FA and DDT in micro-tubes with sub- and near-millimeter radii ${ }^{2-24}$. At the same time, these experiments ${ }^{13,14}$ have demonstrated some features of flame dynamics different from the theoretical predictions ${ }^{19,20}$, such as a linear regime of FA instead of the exponential one. Subsequent numerical study ${ }^{21}$ has explained such a discrepancy by the influence of gas compression neglected in Refs. 19,20, which moderate the acceleration process and make it linear for sufficiently large values of the flow Mach number, see also Ref. 25 for the theoretical explanation of this effect. By the end of the FA process in channels with adiabatic walls, the flame propagation speed saturates to the Chapman-Jouguet deflagration speed ${ }^{11,26,27}$, unless an explosion of the fuel mixture happens earlier. Another important observation of the experiments ${ }^{13,14}$ is a rather surprising possibility of combustion failure at the end of a powerful process of flame acceleration in some cases ${ }^{13}$. This effect, which is presumably related to thermal losses to the channel walls, has not been studied in detail so far.

Thermal losses to the walls, which are inevitably present in any experiment on FA in tubes, are expected to play an important role in micro-channels by working against the acceleration mechanism. For example, in channels with isothermal walls maintained at a temperature equal to the initial one, the thermal losses eventually cool the burnt gas down to the wall temperature, thus effectively reducing the effect of thermal expansion as the key element of the flame acceleration mechanism. In spite of obvious importance of the wall thermal losses, relatively few works have addressed the influence of this effect on FA in tubes. One of the earliest simulation runs on the flame dynamics in a channel with isothermal walls is presented in Ref. 23, where pulsations of the burning velocity have been observed for combustion in a narrow channel with $\operatorname{Re} \sim 10$. These pulsations have been interpreted in Ref. 23 as a footprint of a regular oscillating regime of flame propagation similar to the flame oscillations in open adiabatic channels obtained later in Ref. 28 The simulations ${ }^{23}$ have been refined in Ref. 29 with the same conclusions about the flame dynamics. The oscillatory flame dynamics in the presence of heat losses at the walls also has been observed in the recent simulations of DDT in narrow channels ${ }^{30,31}$.
In the presence of strong thermal losses at the walls, one may question the very possibility of FA. Indeed, one should naturally expect a dominating role for thermal losses in sufficiently narrow channels, which may not only terminate FA, but even lead to complete extinction of the burning process; this problem is of special importance for micro-combustion applications ${ }^{32,33}$. Moderation of FA due to heat loss has been identified numerically in Ref. 34, where the possibility of flame extinction after initial acceleration period has been found as well. In wide channels, with the increase of the flame propagation Reynolds number, the role of thermal losses decreases, but the FA mechanism becomes weaker too ${ }^{19,20}$, and it remained unclear which of these two effects prevails.

Simulations of DDT in channels with cold isothermal walls were performed in Refs. 35-37, where the stage of explosion and detonation onset was discussed to a larger extent, and FA stage was scrutinized to a lesser extent. For that purpose, in most simulations the second order (with respect to density) Arrhenius reaction was chosen, which is more favorable for explosion onset, as compared to the first order reaction kinetics ${ }^{35}$

In the present work, we numerically investigate the influence of thermal losses on flame quenching dynamics in channels with cold isothermal walls. We take realistically low initial values of the Mach number associated with flame propagation, $M_{0} \equiv S_{L} / c_{s} \sim 10^{-3}$ (where $c_{s}$ is the speed of sound in the fuel mixture), which is characteristic, e.g., for hydrocarbon-air flames. The size of the initial flame embryo is taken small enough to mimic small ignition energy, but is larger than a quenching distance in order to successfully initiate self-sustained flame propagation. For most choices of the problem's parameters, we obtain initial FA after ignition at the closed channel end, which resembles qualitatively the adiabatic case $^{19,20}$, but develops noticeably slower, in an approximately linear regime instead of the exponential one and persists only for a limited time interval. At the same time, the linear regime is consistent with experimental observations ${ }^{13,14}$, which inevitably involve thermal losses to the walls. Subsequently, heat loss to the walls reduces the temperature and hence the volume of the burnt gas behind the flame front, which produces a reverse flow in the direction of the closed channel end. When the amount of the burnt gas becomes sufficiently large, the reverse flow stops the acceleration process and drives the flame backwards, with modifications of the flame front shape from convex to concave. Eventually, the flame extinguishes. Qualitatively, this scenario reproduces the possible combustion failure during DDT observed experimentally in Refs. 13,14. The effect of the flame propagation Reynolds number on initial FA is investigated. 


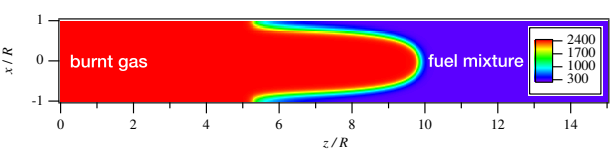

FIG. 1. (Color online) Temperature distribution (in K) for a flame propagating from the closed end $(z=0)$ with adiabatic walls, with $\operatorname{Re}=10$ and $\Theta=8$ at the time instant $S_{L} t / R=$ 0.5 , see the text for other simulation parameters.

\section{BASIC EQUATIONS AND GEOMETRY OF THE PROBLEM}

We consider a flame front propagating in a long twodimensional (2D) channel from the closed end to the open one as illustrated in Fig. 1 for the case of adiabatic walls. The problem formulation is quite similar to that of Ref. 19, except for cold isothermal walls of the channel employed here instead of the adiabatic ones investigated in Ref. 19, thus focusing on the influence of thermal losses to the walls on FA. The case of adiabatic walls will be also considered for the sake of comparison. We study the flame dynamics by using high-fidelity laminar numerical simulations of the Navier-Stokes combustion equations

$$
\begin{gathered}
\frac{\partial \rho}{\partial t}+\frac{\partial}{\partial x_{i}}\left(\rho u_{i}\right)=0, \\
\frac{\partial}{\partial t}\left(\rho u_{i}\right)+\frac{\partial}{\partial x_{j}}\left(\rho u_{i} u_{j}+\delta_{i j} p-\gamma_{i j}\right)=0, \\
\frac{\partial}{\partial t}\left(\rho \varepsilon+\frac{1}{2} \rho u_{i} u_{i}\right)+ \\
\frac{\partial}{\partial x_{j}}\left(\rho u_{j} h+\frac{1}{2} \rho u_{i} u_{i} u_{j}+q_{j}-u_{i} \gamma_{i j}\right)=0, \\
\frac{\partial}{\partial t}(\rho Y)+\frac{\partial}{\partial x_{i}}\left(\rho u_{i} Y-\frac{\zeta}{\mathrm{Sc}} \frac{\partial Y}{\partial x_{i}}\right)=-\Omega,
\end{gathered}
$$

where $\rho$ is the density, $Y$ is the mass fraction of the fuel mixture, $\varepsilon=Q Y+C_{V} T$ and $h=Q Y+C_{P} T$ are the specific internal energy and enthalpy, $Q$ is the specific energy release in the reaction, $C_{V}, C_{P}$ are the heat capacities at constant volume and pressure. We consider a single irreversible Arrhenius reaction of the first order with the activation energy $E_{a}$ and the constant of time dimension $\tau_{R}$,

$$
\Omega=\frac{\rho Y}{\tau_{R}} \exp \left(-E / R_{p} T\right) .
$$

The stress tensor $\gamma_{i j}$ and the energy diffusion vector $q_{j}$ are

$$
\gamma_{i j}=\zeta\left(\frac{\partial u_{i}}{\partial x_{j}}+\frac{\partial u_{j}}{\partial x_{i}}-\frac{2}{3} \frac{\partial u_{k}}{\partial x_{k}} \delta_{i j}\right)
$$

$$
q_{j}=-\zeta\left(\frac{C_{p}}{\operatorname{Pr}} \frac{\partial T}{\partial x_{j}}+\frac{Q}{\operatorname{Sc}} \frac{\partial Y}{\partial x_{j}}\right),
$$

where $\zeta \equiv \rho \nu$ is the dynamic viscosity, $\operatorname{Pr}$ and Sc are the Prandtl and Schmidt numbers, respectively. The gas mixture is an ideal gas of a constant molecular weight $m=2.9 \times 10^{-2} \mathrm{~kg} / \mathrm{mol}$, with $C_{V}=5 R_{p} / 2 m, C_{P}=$ $7 R_{p} / 2 m$, where $R_{p} \approx 8.31 \mathrm{~J} /(\mathrm{mol} \mathrm{K})$ is the universal gas constant. The equation of state for an ideal gas is

$$
P=\rho R_{p} T / m \text {. }
$$

We consider a 2D channel of half-width $R$ with non-slip boundary conditions, $\mathbf{u}=0$, at the top, bottom and left (closed end) walls. Both isothermal cold walls, $T=T_{f}$, where $T_{f}$ is temperature of the fuel mixture, and adiabatic walls, $\hat{\mathbf{n}} \cdot \nabla T=0$, where $\hat{\mathbf{n}}$ is a normal vector at the wall, are considered and compared. We take the initial pressure and temperature of the fuel mixture corresponding to the normal conditions, $P_{f}=10^{5} \mathrm{~Pa}$ and $T_{f}=300 \mathrm{~K}$, with the thermal and chemical parameters of the fuel mixture chosen to reproduce the most important properties of methane and propane laboratory flames. The dynamic viscosity $\zeta=1.7 \times 10^{-5} \mathrm{~N} \mathrm{~s} / \mathrm{m}^{2}$, and the Prandtl number $\operatorname{Pr}=1$. To avoid the thermaldiffusion instability we employ the unity Lewis number, $\mathrm{Le} \equiv \mathrm{Sc} / \mathrm{Pr}=1$. The activation energy is $E_{a}=32 R_{p} T_{f}$. We take the planar flame velocity $S_{L}=34.7 \mathrm{~cm} / \mathrm{s}$ corresponding to the initial Mach number $\mathrm{Ma}=10^{-3}$. The flame thickness in our calculations is defined conventionally as

$$
L_{f} \equiv \frac{\nu}{\operatorname{Pr} S_{L}},
$$

then the Reynolds number associated with the flame speed, Re $\equiv S_{L} R / \nu$, indicates the channel half-width scaled by the flame thickness

$$
\operatorname{Re}=\frac{R}{\operatorname{Pr} L_{f}} .
$$

Still, we would like to stress that the flow Reynolds number may be much higher due to FA. Thermal expansion in the burning process is determined by the energy release in the reaction and characterizes the density ratio of the fuel mixture to the burnt gas, $\Theta \equiv \rho_{f} / \rho_{b}$; we take $\Theta=8$, typical for methane and propane burning as the main case of the numerical studies, and then compare the results to the cases of larger, $\Theta=10$, and smaller, $\Theta=5$, density ratios.

We use a 2D in-house code that is based on a Volvo Aero finite-volume solver ${ }^{38}$; the code is robust, and it was utilized successfully in studies of laminar burning, hydrodynamic flame instabilities, development of corrugated flames, flame acceleration, and related phenomena. The numerical scheme and the computational methods were described in detail in our previous papers, see, e.g., Refs. 11,39-42. Isothermal boundary condition was tested in Refs. 43-45. In the present simulations, we consider different channel widths in the range $5 L_{f}<2 R<40 L_{f}$, 
and take the length of the channel much bigger than its width, $\sim(100-1000) R$. At such large values, variations of the channel length did not influence the simulation results. We use a rectangular orthogonal mesh described in detail in Ref. 11. To perform the calculations in a reasonable time, the mesh was made non-uniform along the $z$-axis, with a flame mesh sub-domain having a constant grid size of $0.2 L_{f}$, which allows resolving the internal flame structure well. Grid independence tests are presented in the Appendix. Outside of the region of fine grid, the mesh size grows gradually in the $z$-direction, with $5 \%$ change in size between the neighbouring cells; we successfully employed the same method ${ }^{19}$ to study FA in channels with adiabatic walls. Along the $x$-axis we used a uniform grid. Here we stress that $L_{f}$, as given by Eq. (9), is just a mathematical parameter of length dimension, while the realistic flame thickness is about an order of magnitude larger ${ }^{28}$. By using such a mesh, we are able to resolve the zone of large velocity gradients close to the walls. Similar to Ref. 19, we use the pseudo Zeldovich - Frank-Kamenetskii (ZFK) solution for a planar flame front ${ }^{2}$ as an initial condition. The planar flame front is initiated at a distance $6 L_{f}$ from the closed channel end. We use non-reflecting boundary conditions at the open end of the channel, as described in Ref. 19, to avoid reflections of weak shocks and sound waves from the open end, which otherwise might influence burning and the process of FA.

\section{SIMULATION RESULTS AND DISCUSSION}

For a fixed density ratio $\Theta$, the flame dynamics is controlled by the flame propagation Reynolds number, Eq. (10). In the case of a very narrow channel, $\operatorname{Re}=5$, the initial ignition kernel at the channel end does not lead to flame initiation and gets eventually cooled down by the walls. This result agrees well with the commonly accepted knowledge that a propagating flame stays away from a cold wall with the quenching region being about $6 L_{f}$, see Refs. 46,47 .

The most representative cases for the problem solution correspond to wider channels, $\operatorname{Re}>10$, with characteristic temperature snapshots shown in Fig. 2 for $\mathrm{Re}=10$ at the time instants $S_{L} t / R=0-3.5$, with equal time intervals of $0.5 R / S_{L}$. In that case, a self-sustained flame is ignited and initially self-accelerates as demonstrated by the snapshots for $S_{L} t / R=0-1.5$. Then the flame speed slows down, until the flame stops and starts moving backward towards the closed channel end. The backward motion is accompanied by modifications of the flame front shape, from the convex to a concave one.

Figure 3 presents the propagation speed of the flame front at the central line of the channel for the same case as in Fig. 2. Initial FA, observed approximately for $S_{L} t / R \leq 1.5$, is qualitatively similar to the adiabatic case, also presented in Fig. 3 for comparison, for the same $\operatorname{Re}=10$ and $\Theta=8$. Still, the adiabatic case exhibits

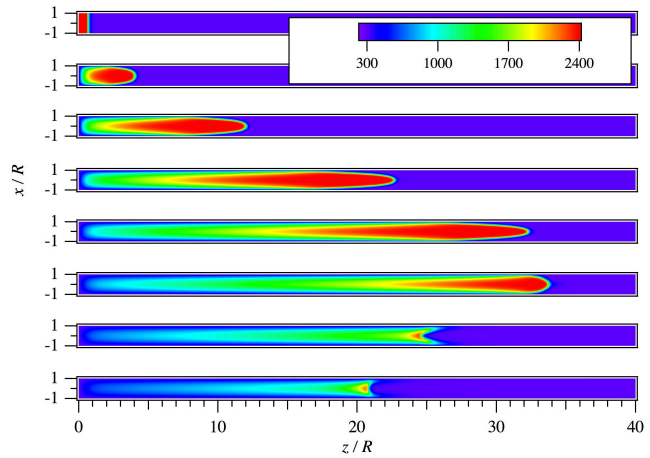

FIG. 2. (Color online) Temperature snapshots (in K) for a flame in a channel with cold isothermal walls for $\mathrm{Re}=10$ and $\Theta=8$ at the time instants $\tau=S_{L} t / R=0-3.5$, with equal time intervals of 0.5 .

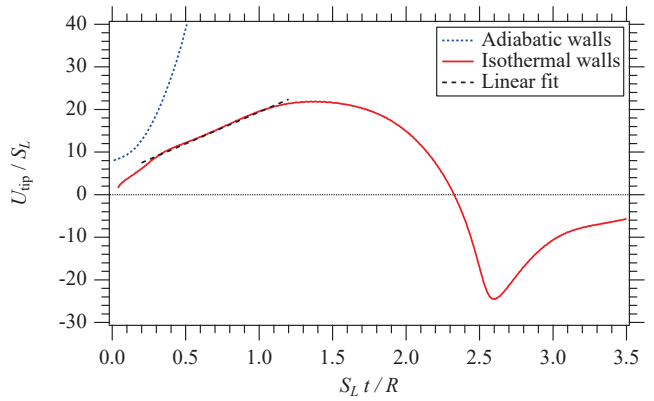

FIG. 3. (Color online) Propagation speed of the flame front at the channel center-line (the flame tip velocity) versus the scaled time $S_{L} t / R$ for $\operatorname{Re}=10, \Theta=8$ for isothermal and adiabatic walls. The straight line shows the linear fit used to calculate the flame acceleration.

powerful exponential flame acceleration

$$
\frac{U_{\text {tip }}}{S_{L}} \propto \exp \left(\sigma S_{L} t / R\right)
$$

where $\sigma$ is the scaled acceleration rate, in agreement with the previous work ${ }^{19}$, where the exponential acceleration regime was predicted theoretically and validated numerically. Unlike the adiabatic case, in the channel with cold walls the flame front accelerates initially in an approximately linear manner, which may be described as

$$
\frac{U_{\mathrm{tip}}}{S_{L}} \approx a \frac{S_{L} t}{R}+\mathrm{const}
$$

where $a$ is the scaled acceleration. Note the noticeably smaller initial flame tip velocity in the case of cold walls. For $1.5 \leq S_{L} t / R \leq 2.3$ initial FA in a channel with 


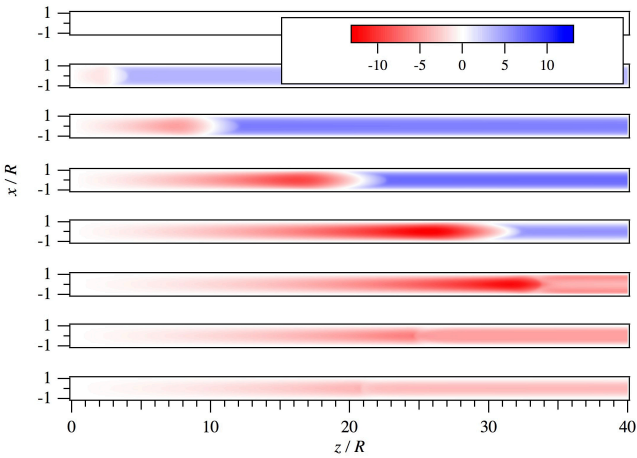

FIG. 4. (Color online) Snapshots of the gas velocity field $u_{z} / S_{L}$ for a flame in a channel with cold isothermal walls for $\operatorname{Re}=10$ and $\Theta=8$ at the time instants $\tau=S_{L} t / R=0-3.5$ with equal time intervals. The time instant are taken the same as in Fig. 2.

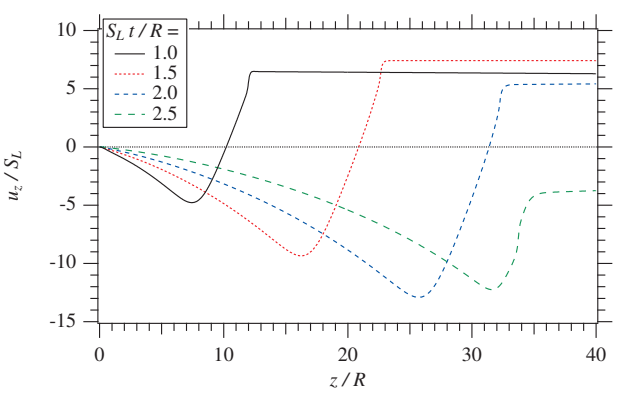

FIG. 5. (Color online) Gas velocity profile in the center line $(x=0)$ of a channel with cold isothermal walls for $\mathrm{Re}=10$ and $\Theta=8$ at the time instants $S_{L} t / R=1.0 ; 1.5 ; 2.0 ; 2.5$.

cold walls is followed by flame deceleration, see Fig. 3 Subsequently, we observe a negative flame velocity (i.e. backward flame propagation) for $S_{L} t / R>2.3$. Flame extinction occurs approximately at $S_{L} t / R \approx 4.0$.

The qualitative difference in the long-time flame dynamics between the isothermal and adiabatic channels in Figs. 1-3 is related to relaxation of the burnt gas temperature down to the wall temperature because of the heat losses to the isothermal cold walls. In addition, we also observe incomplete burning by the side walls, with the quenching distance between the flame "skirt" and the walls being $\sim 4 L_{f}$, which is in reasonable agreement with Refs. 46,47. Still, incomplete burning does not seem to play an important role in the flame dynamics. As one of the main effects in the process, cooling down of the burnt gas reduces the gas volume, which leads to a reverse flow of the burnt gas towards the closed channel end. Modifications of the flow velocity in the burning process is pre-
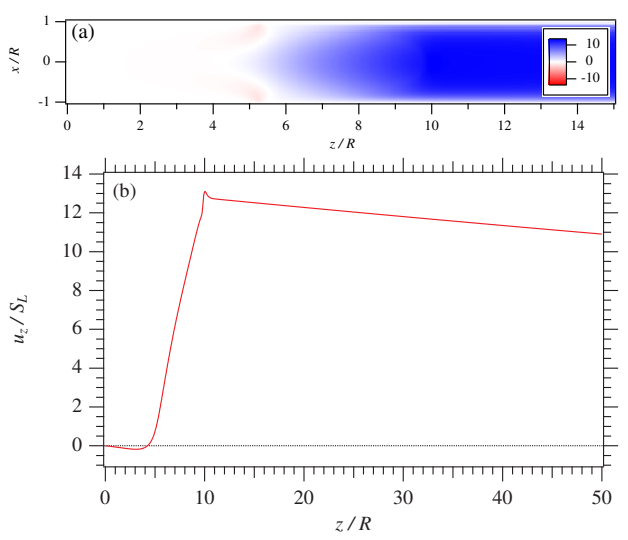

FIG. 6. (Color online) (a) Velocity field $u_{z} / S_{L}$ and (b) velocity profile at the center line $x=0$ for a flame propagating from the closed channel end $z=0$ with adiabatic walls, with $\operatorname{Re}=10$ and $\Theta=8$ at the time instant $S_{L} t / R=0.5$, same as for Fig. 1.

sented in the $u_{z}$-velocity snapshots in Fig. 4, corresponding to the same time instants as the temperature snapshots in Fig. 2, while Fig. 5 shows the velocity profile at the center-line of the channel for different time instants. At the initial stage of FA, $S_{L} t / R \leq 1.5$, Fig. 4 demonstrates a strong flow of the fuel mixture towards the open end, which is the key element of the FA mechanism. Simultaneously, we observe emergence of the reverse flow in the burnt gas, , directed towards the closed end and produced due to heat losses. Initially, the reverse flow is noticeably weaker than the main flow in the fuel mixture, and the flame accelerates. However, the strength of the reverse flow is determined by the total amount of the burnt gas. As the burnt gas is accumulated behind the flame front, the reverse flow becomes stronger; the strengths of the main and the reverse flows are approximately the same by the scaled time $S_{L} t / R \approx 1.5$, when FA is terminated and followed by deceleration; still, the flame keeps moving forward for a while. Eventually, the cooling process becomes so strong, that the reverse flow overwhelms completely and the fuel mixture starts moving towards the closed tube end along with the burnt gas. This happens approximately for $S_{L} t / R \geq 2.3$, when the flame front is drifted backwards by the strong reverse flow. Since the maximum gas velocity (by absolute value) of the reverse flow is attained at the channel center line, the flame shape is naturally changed from convex to concave, which looks formally similar to the famous "tulip" flame phenomenon ${ }^{48-52}$. For comparison, Fig. 6 shows the $u_{z}$-velocity snapshot and the gas velocity profile for the channel center-line for the adiabatic Re $=10$ and $\Theta=8$ case at the same time instant as in Fig. 1. Unlike the isothermal channel, the adiabatic case demonstrates 


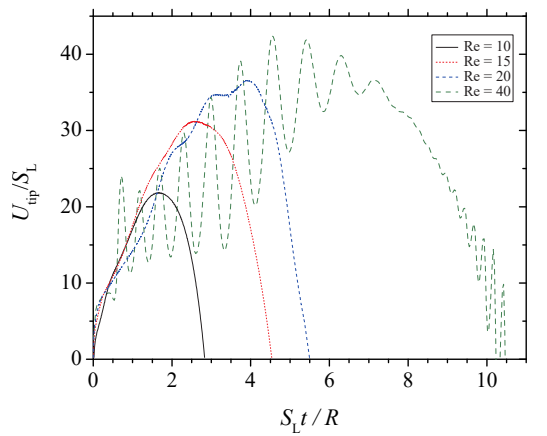

FIG. 7. (Color online) Propagation speed of the flame front at the channel center-line (the flame tip) versus the scaled time $S_{L} t / R$ for $\operatorname{Re}=10-40, \Theta=8$, for cold isothermal walls.

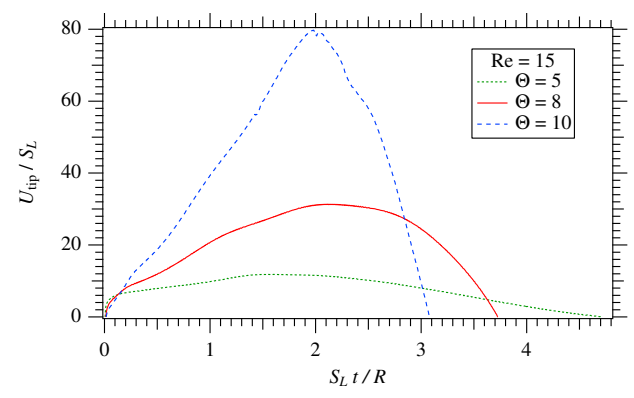

FIG. 8. (Color online) Propagation speed of the flame front at the channel center-line (the flame tip) versus the scaled time $S_{L} t / R$ for $\operatorname{Re}=15$ and $\Theta=5 ; 8 ; 10$ for cold isothermal walls.

strong gas velocity in the fuel mixture with almost zero reverse flow in the burnt gas; such a velocity distribution produces powerful exponential acceleration of the flame front in the adiabatic channel.

We have performed simulation runs similar to those of Fig. 3 for different values of the density ratio, $\Theta=$ $5 ; 8 ; 10$, with the flame Reynolds numbers in the range Re $=10-40$. In all cases we observed qualitatively the same flame dynamics with initial acceleration followed by deceleration and then by extinction, see Figs. 7 and 8 . For sufficiently wide channels, $\operatorname{Re} \geq 40$, the acceleration process was accompanied by relatively short-period oscillations of the flame front, qualitatively similar to Ref. 28 with the characteristic oscillation period of $\sim R / S_{L}$ Still, these oscillations have not modified the overall picture of the flame dynamics. According to Fig. 7, the maximum speed of the flame tip attained in the acceleration process grows with the flame Reynolds number,

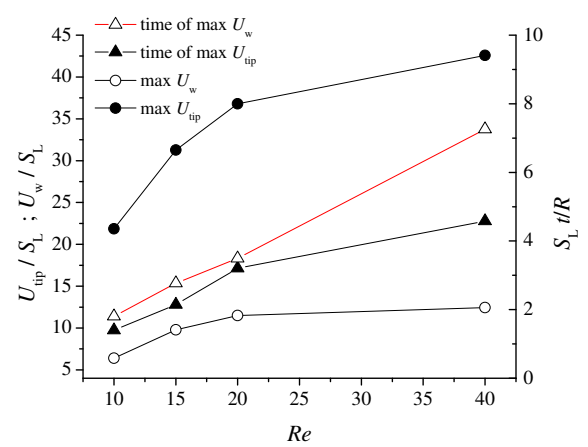

FIG. 9. (Color online) Maximum propagation speed of the flame front at the channel center-line (the flame tip), $U_{\text {tip }}$, maximum burning rate $U_{w}$, and corresponding scaled times needed to attain the maxima, as a function of the Reynolds number $\operatorname{Re}=10-40$, for $\Theta=8$.

i.e., for wider channels, and it takes longer (scaled) time for the flame to reach the maximum speed. The results for the maximum tip velocity for $\Theta=8$ are presented in Fig. 9 versus the Reynolds number, together with the maximum burning rate, calculated as ${ }^{53}$

$$
U_{w}=\frac{1}{2 R \rho_{f}} \iint \Omega d x d z,
$$

with the reaction rate $\Omega$ given by Eq. (5). The respective results for the scaled time needed to attain the maximum propagation speed are also shown in Fig. 9. It is seen that the maximum propagation speed $U_{t} i p$, the maximum burning rate $U_{w}$, and the corresponding scaled times needed to attain their maxima, all grow with $R e$.

We have also investigated the value of the scaled flame acceleration in channels with isothermal walls at the initial stage of the burning process. The respective numerical results are summarized in Fig. 10, where we plot the slope $a_{w}$ of the initial acceleration of the burning rate,

$$
\frac{U_{w}}{S_{L}} \approx a_{w} \frac{S_{L} t}{R}+\text { const. }
$$

Here we stress one more time that FA in the channels with isothermal walls develops approximately in the linear regime of Eq. (12) in contrast to the exponential regime of Eq. (11) inherent to the adiabatic case. We point out that the experiments on ethylene-oxygen FA in micro-channels ${ }^{13,14}$ have also reported a linear acceleration trend. Previous numerical simulations ${ }^{21,22}$ of the developed stages of FA in adiabatic channels have attributed such a transition from exponential to linear acceleration to the gas compressibility effects and the Mach number of the flow approaching unity. Besides, linear FA has been encountered in numerical simulations of burning 


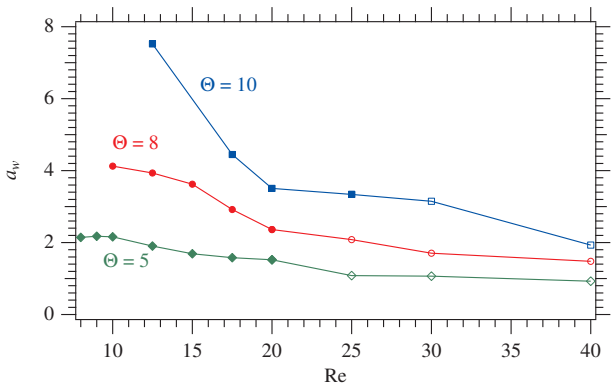

FIG. 10. (Color online) Slope $a_{w}$ of the burning rate $U_{w}$, Eq. (14), for the initial acceleration (see Fig. 3) versus the Reynolds number, for cold isothermal walls. Open symbols correspond to cases where oscillations are present.

in adiabatic channels for a sufficiently small density ratio or sufficiently large channels ${ }^{54}$. In case of isothermal walls with temperature higher than the initial temperature of fresh fuel mixture, FA in channels with non-slip at the walls may be near-linear as well ${ }^{55}$. The present simulations demonstrate that the linear acceleration regime may also appear because of heat losses. By comparing all these cases, one can presumably discuss a common tendency of the linear flame acceleration regime replacing the exponential one as soon as the acceleration mechanism weakens for any reason. One is tempted to treat linear FA, Eq. (12), as the first term in the Taylor expansion for weak exponential acceleration, Eq. (11),

$$
\frac{U_{\text {tip }}}{S_{L}} \propto \exp \left(\sigma S_{L} t / R\right) \approx 1+\sigma \frac{S_{L} t}{R},
$$

with a small acceleration rate $\sigma \ll 1$. Still, one has to be cautious with such a treatment as the linear acceleration regime has been observed up to the instants with noticeable increase of the flame propagation speed, when the term $a S_{L} t / R$ is not small anymore.

As demonstrated by Fig. 10, FA in channels with cold walls intensifies with the density ratio $\Theta$ and weakens with the channel width, i.e., with $R e$. These tendencies are qualitatively the same as those identified earlier for FA in channels with adiabatic walls ${ }^{19}$. We remind here that the scaled acceleration rate in sufficiently wide channels with adiabatic walls may be described by a simplified analytical formula $\sigma=\Theta^{2} / \mathrm{Re}^{19}$, which reveals the most important tendencies of the process.

In narrow channels with a heat loss at the walls, the flame dynamics is expected to be effected both by the viscosity and the thermal conductivity. In this light, we discuss the effect of Prandtl number on flame dynamics. Fig. 11 shows the time evolution of the burning rate $U_{w}$ for a constant $R e=15$, and for three different Prandtl numbers $\operatorname{Pr}$ in the range between 0.5 and 1 . At lower values of $\operatorname{Pr}$, a possibility of successful ignition is observed: after initial flame accelelation and deceleration, the flame

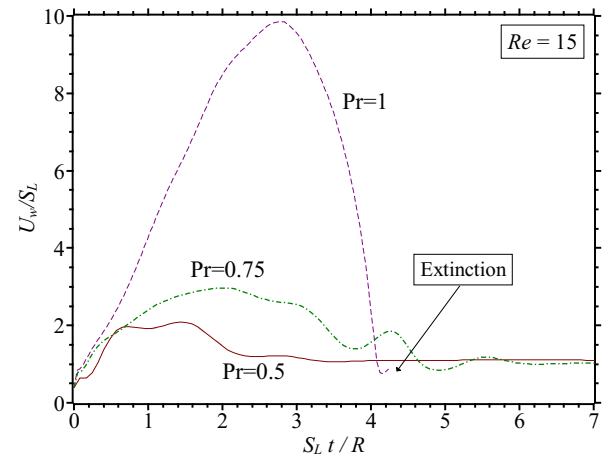

FIG. 11. (Color online) Time evolution of the burning rate $U_{w}$ for a constant $R e=15$, and for various Prandtl numbers Pr. Case with extinction is indicated with an arrow.

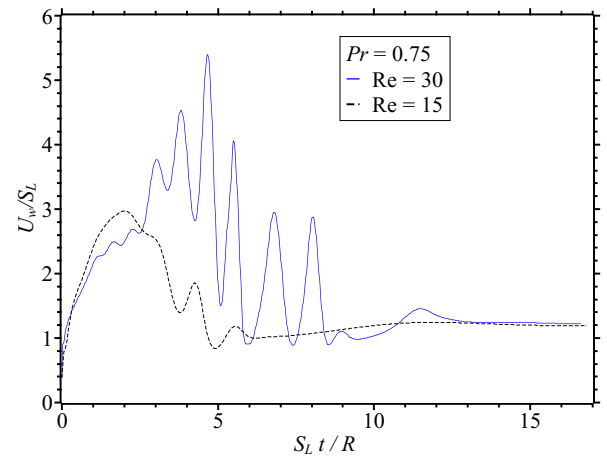

FIG. 12. (Color online) Time evolution of the burning rate $U_{w}$ for constant $\operatorname{Pr}=0.75$, and two different $\operatorname{Re}=15,30$.

burning rate attains a constant value slightly above $S_{L}$. This result indicates that at certain channel widths $2 R$ and flame Reynolds numbers Re, a lower Pr may be favorable for a successful ignition. Fig. 12 shows the time evolution of the burning rate $U_{w}$ for constant $\operatorname{Pr}=0.75$, and two different $\mathrm{Re}=15$ and 30 . The burning rate evolution undergoes much stronger oscillations for $\mathrm{Re}=30$, which is in line with flame tip velocity behavior shown in Fig. 7, but, contrary to $\operatorname{Pr}=1$ cases of Fig. 7, here we observe successful ignition.

Finally, it is interesting to compare the present simulation results to the experimental observations ${ }^{13}$. Although heat losses to the walls has not been directly investigated in the experiments ${ }^{13,14}$, some heat losses are inevitable in realistic combustion in channels. Of course, the present simulation runs employ noticeably lower flame propagation Reynolds numbers, $\operatorname{Re}_{\text {num }} \leq 40$, than $\operatorname{Re}_{\exp } \approx 100$ in the experiments ${ }^{13}$, thereby making the comparison 
rather qualitative than quantitative. Keeping in mind that the laminar burning velocity of the ethylene flames in the experiments ${ }^{13}$ is $S_{L} \approx 5.5 \mathrm{~m} / \mathrm{s}$, the present simulation results suggest the maximum flame speed attained in the acceleration process to be $\max \left(U_{\text {tip }}\right) \sim 220 \mathrm{~m} / \mathrm{s}$, which is comparable to the speed of sound. This is a noticeably smaller value than the maximum deflagration speed $\approx 1500 \mathrm{~m} / \mathrm{s}$ observed in the experiments ${ }^{13}$. Still, we point out that (1) the maximum flame speed attained in the process of FA increases with the flame Reynolds number; (2) cylindrical tubes employed in the experiments are expected to provide much stronger FA as compared to the present computational case of $2 \mathrm{D}$ channels see Refs. 19,20, and (3) that the wall boundary conditions in the experiments do not necessarily correspond to the isothermal cold walls, but represent an intermediate case between the adiabatic and isothermal ones.

In the recent experiments with ethylene-oxygen flames in a millimeter-wide channel ${ }^{56}$, which are to some extent geometrically more similar to the present simulations, the flame acceleration was quantified. It is noted that for the fast burning ethylene-oxygen mixtures in narrow channels, pressure waves play a major role in flame dynamics. Before the onset of the leading shock wave, i.e. when the effects of gas compression ahead of the flame are minor, the experimentally obtained non-dimensional flame tip acceleration $a_{t i p} R / S_{L}^{2}$ is of the order of 200 In the present simulations, the largest flame acceleration of around 40 is attained for $\Theta=10, \operatorname{Re}=15$, see Fig. 8 . In the present study, the flame and flow Mach numbers are relatively low. The role of gas compression in flame acceleration in channels with heat loss at the walls is an interesting topic for further inquiry.

With some caution, we may state that the present simulation results can be qualitatively compared to the case of Ref. 13, with powerful FA during limited time, leading to a large flame propagation speed and followed by subsequent combustion failure.

\section{SUMMARY}

In the present work, we have investigated flame propagation in long narrow channels, from the closed to the open end, for the case of cold isothermal channel walls. The main focus was on the competition of flame acceleration (FA) and heat losses to the walls, and the corresponding influence of gas expansion and contraction on the flame dynamics and failure. The problem is very important in the context of flame dynamics in narrow channels; in particular, it is needed for better understanding of the experimental results on DDT in microchannels ${ }^{13,14}$. Moreover, the present results may contribute to better understanding of the importance of thermal gas expansion and contraction in flame arrestors ${ }^{3,5}$.

The main conclusion of the present work is that, for the small initial flame embryo, the wall heat losses are sufficiently strong to prevent a powerful exponential FA observed for the adiabatic case ${ }^{19,21}$. In the case of cold walls we find much weaker FA developing in an approximately linear regime during a limited time interval. This initial FA happens according to the Shelkin mechanism due to expansion of the burnt gas and nonslip boundary conditions at the walls. Still, heat losses to the cold isothermal walls lead to the temperature relaxation of the burnt gas down to the wall temperature, which creates a reverse flow in the burnt gas towards the closed channel end, working against FA. As the amount of the burnt gas accumulates, the reverse flow becomes stronger, the acceleration process eventually stops, and the flame front is drifted backwards to the closed channels end. Together with the backward motion, the flame shape changes from convex to concave, and combustion failure is observed similar to some of the experimental cases reported previously ${ }^{13}$.

Still, even though limited in time, this initial flame acceleration may considerably promote the flame propagation velocity as compared to the laminar flame speed. For the investigated range of the channel widths (flamerelated Reynolds numbers) the flame speed may increase by a factor of 40 , which is equivalent to near-sonic speed when compared to the experiments on ethylene-air flames ${ }^{13}$. We have investigated the flame dynamics and found that the initial scaled acceleration of the flame tip decreases with the Reynolds number of the flow for channels with cold isothermal walls; this tendency is qualitatively similar to the case of adiabatic channel walls studied previously. At the same time, the maximum flame propagation speed attained in the acceleration process increases with the scaled channel width (and with the flame propagation Reynolds number).

\section{ACKNOWLEDGEMENTS}

The co-authors acknowledge the major contributions by Vitaly Bychkov, who passed away while this work was in progress. D.V. is grateful to Florian Schmidt for the help with computations and to Kun Zhang for useful discussions. The work at Umeå University was supported by the Swedish Research Council and by the Kempe Foundation. The work at Tsinghua University was supported by the National Natural Science Foundation of China (NSFC) through the grant number 51750110503 , and by the State Key Laboratory of Explosion Science and Technology (Beijing Institute of Technology) through the open project number KFJJ21$10 \mathrm{M}$. The work at West Virginia University was supported by the U.S. National Science Foundation (NSF) through CAREER Award No. 1554254 (V.A.). The computations and data handling were enabled by resources provided by the Swedish National Infrastructure for Computing (SNIC) at High Performance Computing Center North (HPC2N) partially funded by the Swedish Research Council through grant agreement no. 201805973, under projects SNIC 2016/1-565, SNIC 2017/1- 


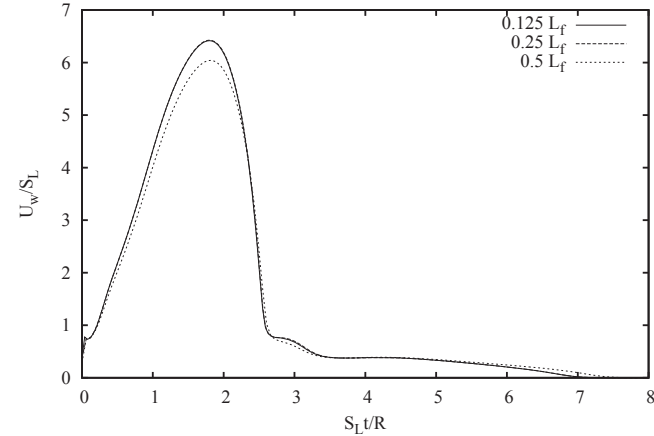

FIG. 13. Scaled burning rate $U_{w} / S_{L}$ versus the scaled time $S_{L} t / R$ for $\operatorname{Re}=10, \Theta=8$ for isothermal walls and grid spacings $0.125 L_{f}, 0.25 L_{f}, 0.5 L_{f}$.

648, SNIC 2018/3-680 and SNIC 2019/3-387. Some pilot results have been presented at 25th International Colloquium on the Dynamics of Explosions and Reactive Systems (ICDERS) held in Leeds, UK, in 2015.

\section{DATA AVAILABILITY STATEMENT}

The data that support the findings of this study are available from the corresponding author upon reasonable request.

\section{APPENDIX: CONVERGENCE TESTS}

We performed resolution tests for $\operatorname{Re}=10, \Theta=8$ to verify that the grid size $0.25 L_{f}$ is sufficient to study the flame acceleration proccess in channel with heat losses. The resolution tests were focused on the temporal evolution of the burning rate, see Fig. 13. The grid size in flame domain varied between $0.125 L_{f}, 0.25 L_{f}$ and $0.5 L_{f}$. The resolution test results are presented in $\mathrm{Ta}-$ ble I and show good convergence of the maximum burning rate, as well as of the instant at which the maximum is attained.

Notation: $\Delta z_{f} / L_{f}$ is the spatial step in the uniform grid domain where burning is localized; $U_{w, \max } / S_{L}$ and $t S_{L} / R$ are the scaled maximum burning rate and the time instant at which the maximum is attained, respectively (see Fig. 13). $\Delta U_{w, \max } / S_{L}$ and $\Delta t S_{L} / R$ are the increments of $U_{w, \max } / S_{L}$ and $t S_{L} / R$ calculated in the table row i as $\Delta U_{w, \max }(i)=U_{w, \max }(i)-U_{w, \max }(i-1)$ and $\Delta t(i)=t(i)-t(i-1)$.

${ }^{1}$ G. D. Roy, S. M. Frolov, A. A. Borisov, and D. W. Netzer, "Pulse detonation propulsion: challenges, current status, and future perspective," Progress in Energy and Combustion Science 30, 545-672 (2004).
${ }^{2}$ C. K. Law, Combustion Physics (New York: Cambridge University Press, 2006)

${ }^{3}$ L.-Q. Wang, H.-H. Ma, Z.-W. Shen, and D.-G. Chen, "Flame quenching by crimped ribbon flame arrestor: A brief review," Process Safety Progress 38, 27-41 (2019).

${ }^{4}$ A. M. Mahuthannan, J. S. Damazo, E. Kwon, W. L. Roberts, and D. A. Lacoste, "Effect of propagation speed on the quenching of methane, propane and ethylene premixed flames between parallel flat plates," Fuel 256, 115870 (2019).

${ }^{5} \mathrm{Y}$. Wan, C. Wang, Q. Li, and X. Luo, "Experimental study of premixed hydrogen-air flame quenching in a channel with the perforated plate," Fuel 263, 116733 (2020).

${ }^{6}$ F. Kodakoglu, S. Demir, D Valiev, and V. Akkerman, "Analysis of gaseous and gaseous-dusty, premixed flame propagation in obstructed passages with tightly placed obstacles," Fluids $\mathbf{5}, 115$ (2020)

${ }^{7}$ F. Kodakoglu and V. Akkerman, "Analytical study of an effect of gas compressibility on a burning accident in an obstructed passage," Physics of Fluids 32, 073602 (2020).

${ }^{8} \mathrm{G}$. Ciccarelli and S. Dorofeev "Flame acceleration and transition to detonation in ducts," Progress in energy and combustion science 34, 499-550 (2008).

${ }^{9}$ S. B. Dorofeev, "Flame acceleration and explosion safety applications," Proceedings of the Combustion Institute 33, 2161-2175 (2011)

${ }^{10}$ M. Kuznetsov, V. Alekseev, I. Matsukov, and S. Dorofeev, "Ddt in a smooth tube filled with a hydrogen-oxygen mixture," Shock waves 14, 205-215 (2005).

${ }^{11}$ D. Valiev, V. Bychkov, V. Akkerman, L.-E. Eriksson, and C. K. Law, "Quasi-steady stages in the process of premixed flame acceleration in narrow channels," Physics of Fluids 25, 096101 (2013).

${ }^{12}$ R. K. Zipf Jr, V. N. Gamezo, K. M. Mohamed, E. S. Oran, and D. A. Kessler, "Deflagration-to-detonation transition in natural gas-air mixtures" Combustion and flame 161, 2165-2176 (2014).

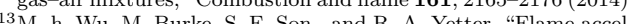
eration and the transition to detonation of stoichiometric ethylene/oxygen in microscale tubes," Proceedings of the Combustion Institute 31, 2429-2436 (2007)

${ }^{14} \mathrm{M} .-\mathrm{H}$. Wu and C.-Y. Wang, "Reaction propagation modes in millimeter-scale tubes for ethylene/oxygen mixtures," Proceedings of the Combustion Institute 33, 2287-2293 (2011).

${ }^{15} \mathrm{M}$.-H. Wu and W.-C. Kuo, "Transition to detonation of an expanding flame ring in a sub-millimeter gap," Combustion and flame 159, 1366-1368 (2012)

${ }^{16}$ D. J. Finigan, B. D. Dohm, J. A. Mockelman, and M. A. Oehlschlaeger, "Deflagration-to-detonation transition via the distributed photo ignition of carbon nanotubes suspended in fuel/oxidizer mixtures," Combustion and Flame 159, 1314-1320 (2012).

${ }^{17} \mathrm{~K}$. Shelkin, "Influence of the wall roughness on initiation and propagation of detonation in gases," Zh. Eksp. Teor. Fiz 10, 823 (1940)

${ }^{18}$ I. Zeldovich, G. I. Barenblatt, V. Librovich, and G. Makhviladze, Mathematical theory of combustion and explosions (Consultants Bureau, New York, NY, 1985)

${ }^{19} \mathrm{~V}$. Bychkov, A. Petchenko, V. Akkerman, and L.-E. Eriksson, "Theory and modeling of accelerating flames in tubes," Physical Review E 72,046307 (2005).

${ }^{20}$ V. Akkerman, V. Bychkov, A. Petchenko, and L.-E. Eriksson, "Accelerating flames in cylindrical tubes with nonslip at the walls," Combustion and Flame 145, 206-219 (2006).

${ }^{21}$ D. M. Valiev, V. Bychkov, V. Akkerman, and L.-E. Eriksson, "Different stages of flame acceleration from slow burning to chapman-jouguet deflagration," Physical Review E 80, 036317 (2009).

${ }^{22}$ L. Kagan and G. Sivashinsky, "The transition from deflagration to detonation in thin channels," Combustion and Flame 134, 389-397 (2003)

${ }^{23}$ J. D. Ott, E. S. Oran, and J. D. Anderson Jr, "A mechanism for flame acceleration in narrow tubes," AIAA journal 41, 1391-1396 


\begin{tabular}{|l|l|l|l|l|}
\hline$\Delta z_{f} / L_{f}$ & $U_{w, \max } / S_{L}$ & $\Delta U_{w, \max } / S_{L}$ & $t S_{L} / R$ & $\Delta t S_{L} / R$ \\
\hline 0.5 & 6.039 & & 1.8184 & \\
0.25 & 6.426 & 0.387 & 1.802 & 0.0164 \\
0.125 & 6.416 & 0.01 & 1.8007 & 0.0013 \\
\hline
\end{tabular}

TABLE I. Resolution tests for $\operatorname{Re}=10, \Theta=8$.

(2003).

${ }^{24}$ D. Valiev, V. Bychkov, V. Akkerman, L.-E. Eriksson, and M. Marklund, "Heating of the fuel mixture due to viscous stress ahead of accelerating flames in deflagration-to-detonation transition," Physics Letters A 372, 4850-4857 (2008).

${ }^{25}$ V. Bychkov, V. Akkerman, D. Valiev, and C. K. Law, "Role of compressibility in moderating flame acceleration in tubes," Physical Review E 81, 026309 (2010).

${ }^{26} \mathrm{R}$. Chue, J. Clarke, and J. Lee, "Chapman-jouguet deflagrations," Proceedings of the Royal Society of London. Series A: Mathematical and Physical Sciences 441, 607-623 (1993).

${ }^{27}$ V. Bychkov, D. Valiev, V. Akkerman, and C. K. Law, "Gas compression moderates flame acceleration in deflagration-todetonation transition," Combustion science and technology 184 1066-1079 (2012)

${ }^{28}$ V. Akkerman, V. Bychkov, A. Petchenko, and L.-E. Eriksson, "Flame oscillations in tubes with nonslip at the walls," Combustion and Flame 145, 675-687 (2006)

${ }^{29} \mathrm{~V}$. N. Gamezo and E. S. Oran, "Flame acceleration in narrow channels: Applications for micropropulsion in low-gravity enviconments," AIAA Journal 44, 329-336 (2006)

${ }^{30}$ J. Huang, W. Han, X. Gao, and C. Wang, "Effects of heat loss and viscosity friction at walls on flame acceleration and deflagration to detonation transition," Chinese Physics B 28, 074704 (2019).

${ }^{31}$ W. Han, J. Huang, G. Gu, C. Wang, and C. K. Law, "Surface heat loss and chemical kinetic response in deflagration-todetonation transition in microchannels," Physical Review Fluid 5, $053201(2020)$

${ }^{32}$ Y. Ju and K. Maruta, "Microscale combustion: Technology development and fundamental research," Progress in energy and combustion science $\mathbf{3 7}, 669-715$ (2011).

${ }^{33}$ N. S. Kaisare and D. G. Vlachos, "A review on microcombustion: Fundamentals, devices and applications," Progress in Energy and Combustion Science 38, 321-359 (2012).

${ }^{34}$ C. Dion, B. Demirgok, V. Akkerman, D. Valiev, and V. Bychkov, "Acceleration and extinction of flames in channels with cold walls," in 25th International Colloquium on the Dynamics of Explosions and Reactive Systems (ICDERS), Leeds, UK (2015).

${ }^{35}$ L. Kagan, D. Valiev, M. Liberman, and G. Sivashinsky, "Effects of hydraulic resistance and heat losses on the deflagration-to detonation transition," in Proceedings of 5th International Colloquium on Pulsed and Continuous Detonations, edited by G. Roy, S. Frolov, and J. Sinibaldy (Torus Press, 2006) pp. 108-112.

${ }^{36} \mathrm{~L}$. Kagan, "On the transition from deflagration to detonation in narrow channels," Mathematical Modelling of Natural Phenomena $2,40-55$ (2007)

${ }^{37}$ L. Kagan and G. Sivashinsky, "On the transition from deflagration to detonation in narrow tubes," Flow, turbulence and combustion 84, 423-437 (2010)

${ }^{38}$ C. Wollblad, L. Davidson, and L.-E. Eriksson, "Large eddy simulation of transonic flow with shock wave/turbulent boundar layer interaction," AIAA journal 44, 2340-2353 (2006).

${ }^{39} \mathrm{~V}$. Akkerman and D. Valiev, "Moderation of flame acceleration in obstructed cylindrical pipes due to gas compression," Physics of Fluids 30, 106101 (2018)
${ }^{40}$ A. Adebiyi, R. Alkandari, D. Valiev, and V. Akkerman, "Effect of surface friction on ultrafast flame acceleration in obstructed cylindrical pipes," AIP Advances 9, 035249 (2019).

${ }^{41}$ A. Adebiyi, E. Ridgeway, R. Alkandari, A. Cathreno, D. Valiev, and V. Akkerman, "Premixed flame oscillations in obstructed channels with both ends open," Proceedings of the Combustion Institute 37, 1919-1926 (2019)

${ }^{42} \mathrm{M}$. Alkhabbaz, O. Abidakun, D. Valiev, and V. Akkerman, "Impact of the lewis number on finger flame acceleration at the early stage of burning in channels and tubes," Physics of Fluids 31, 083606 (2019).

${ }^{43}$ O. J. Ugarte and V. Akkerman, "Computational study of premixed flame propagation in micro-channels with nonslip walls: Effect of wall temperature," Fluids 6, 36 (2021).

${ }^{44} \mathrm{O}$. Abidakun, A. Adebiyi, D. Valiev, and V. Akkerman, "Impacts of fuel nonequidiffusivity on premixed flame propagation in channels with open ends," Physics of Fluids 33, 013604 (2021).

${ }^{45} \mathrm{O}$. J. Ugarte, V. Bychkov, J. Sadek, D. Valiev, and V. AkkerO. J. "Garte, V. Bychkov, J. Sadek, D. Valiev, and V. Akker-
man, "Critical role of blockage ratio for flame acceleration in channels with tightly spaced obstacles," Physics of Fluids 28, 093602 (2016).

${ }^{46}$ C. R. Ferguson and J. C. Keck, "On laminar flame quenching and its application to spark ignition engines," Combustion and Flame 28, 197-205 (1977)

$47 \mathrm{~J}$. Daou and M. Matalon, "Influence of conductive heat-losses on the propagation of premixed flames in channels," Combustion and Flame 128, 321-339 (2002).

${ }^{48}$ C. Clanet and G. Searby, "On the "tulip flame" phenomenon," Combustion and flame 105, 225-238 (1996)

${ }^{49}$ V. Bychkov, V. Akkerman, G. Fru, A. Petchenko, and L.-E. Eriksson, "Flame acceleration in the early stages of burning in tubes," Combustion and Flame 150, 263-276 (2007).

${ }^{50}$ H. Xiao, Q. Wang, X. He, J. Sun, and X. Shen, "Experimental study on the behaviors and shape changes of premixed hydrogenair flames propagating in horizontal duct," International journal of hydrogen energy 36, 6325-6336 (2011)

${ }^{51}$ D. M. Valiev, V. Akkerman, M. Kuznetsov, L.-E. Eriksson, C. K. Law, and V. Bychkov, "Influence of gas compression on flame acceleration in the early stage of burning in tubes," Combustion and flame 160, 97-111 (2013).

${ }^{52}$ H. Wei, X. Zhang, H. Zeng, R. Deiterding, J. Pan, and L. Zhou, "Mechanism of end-gas autoignition induced by flame-pressure interactions in confined space," Physics of Fluids 31, 076106 (2019)

${ }^{53}$ A. Petchenko, V. Bychkov, V. Akkerman, and L.-E. Eriksson, "Flame-sound interaction in tubes with nonslip walls," Combustion and Flame 149, $418-434$ (2007).

${ }^{54}$ B. Demirgok, O. Ugarte, D. Valiev, and V. Akkerman, "Effect of thermal expansion on flame propagation in channels with nonslip walls," Proceedings of the Combustion Institute 35, 929-936 (2015)

${ }^{55}$ M. Baigmohammadi, O. Roussel, and C. M. Dion, "A numerical study of lean propane-air flame acceleration at the early stages of burning in cold and hot isothermal walled small-size tubes," Flow, Turbulence and Combustion 104, 179-207 (2020).

${ }^{56}$ H.-W. Ssu and M.-H. Wu, "Formation and characteristics of composite reaction-shock clusters in narrow channels," Proceedings of the Combustion Institute (2020), 10.1016/j.proci.2020.07.069. 


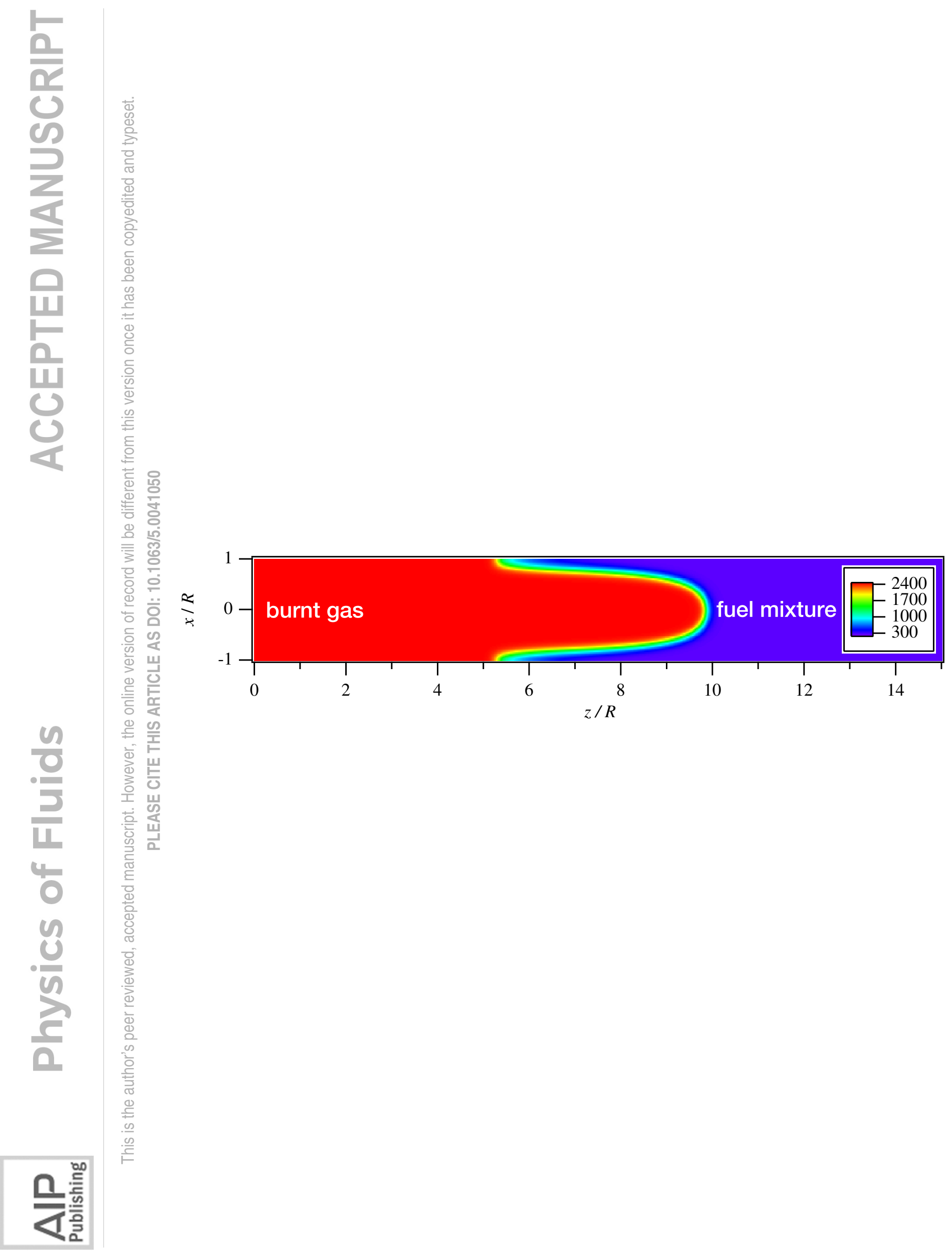




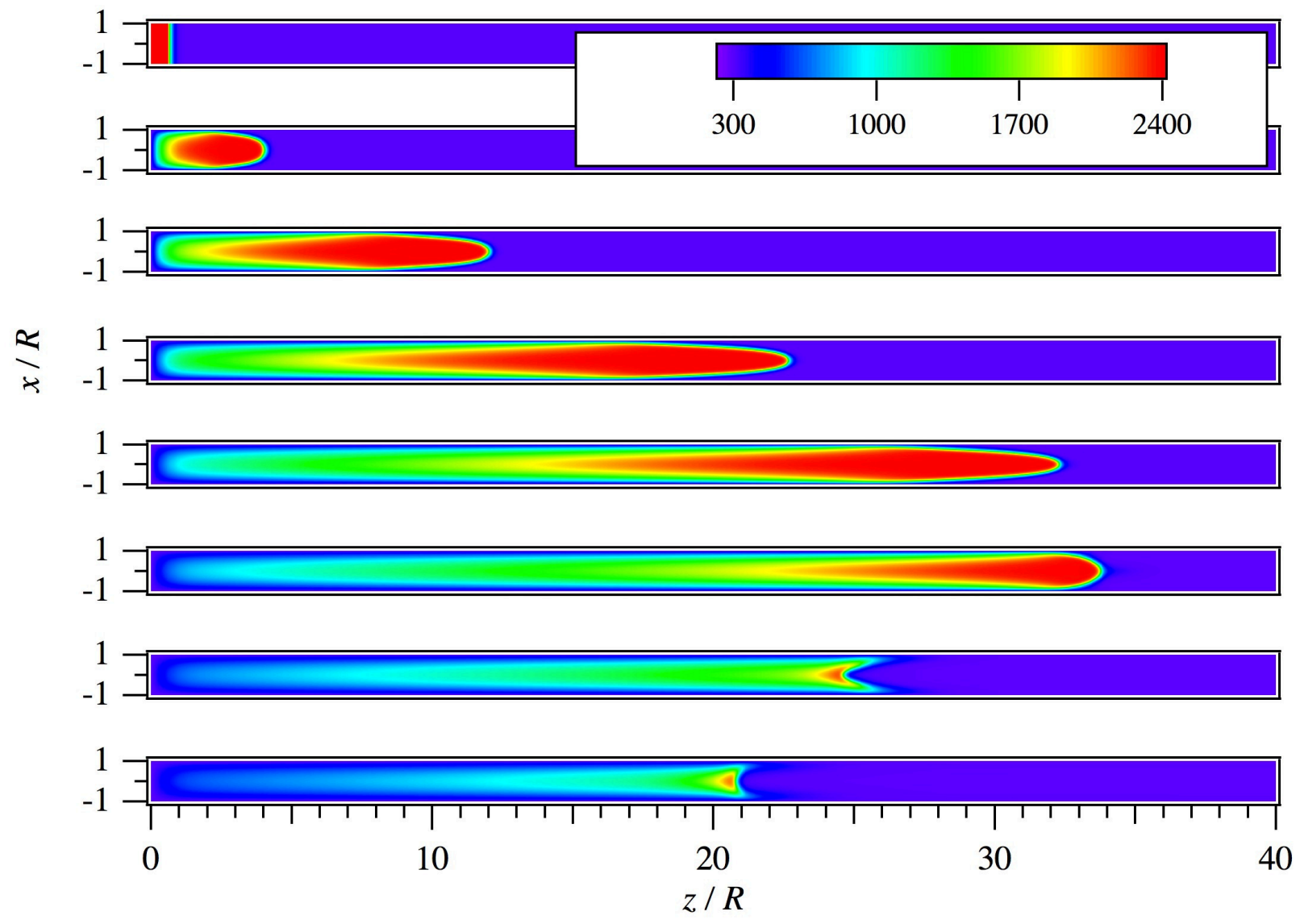




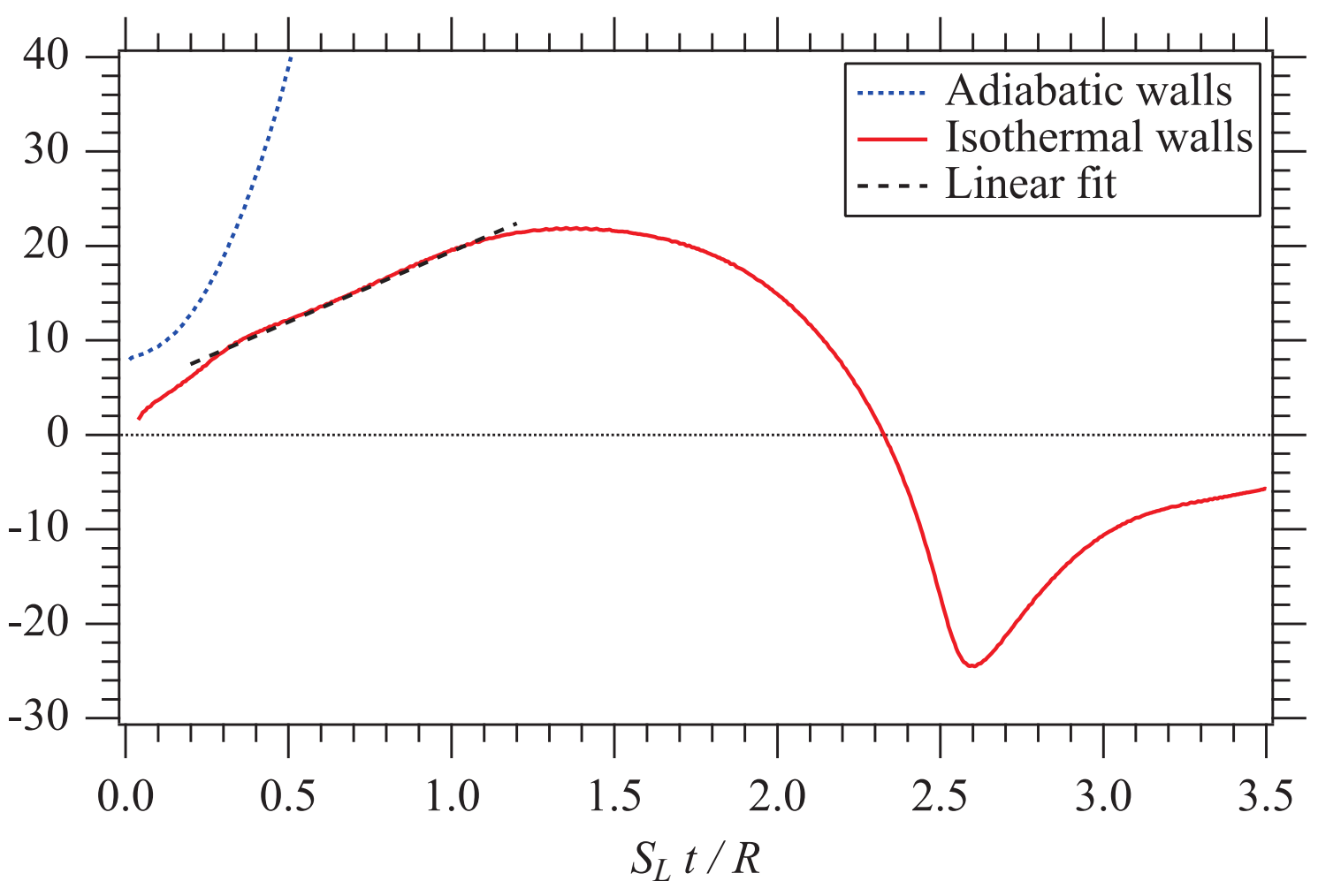




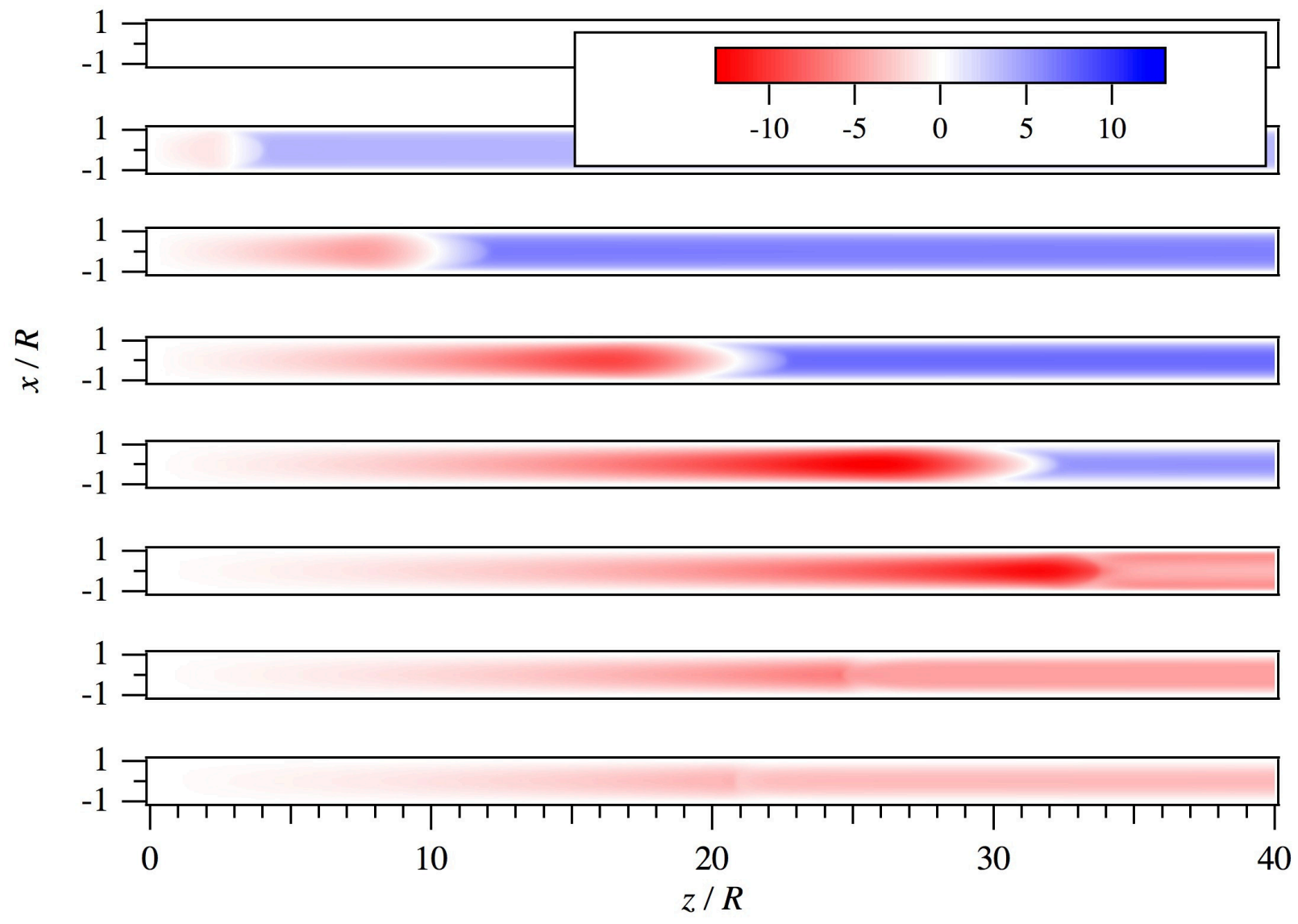




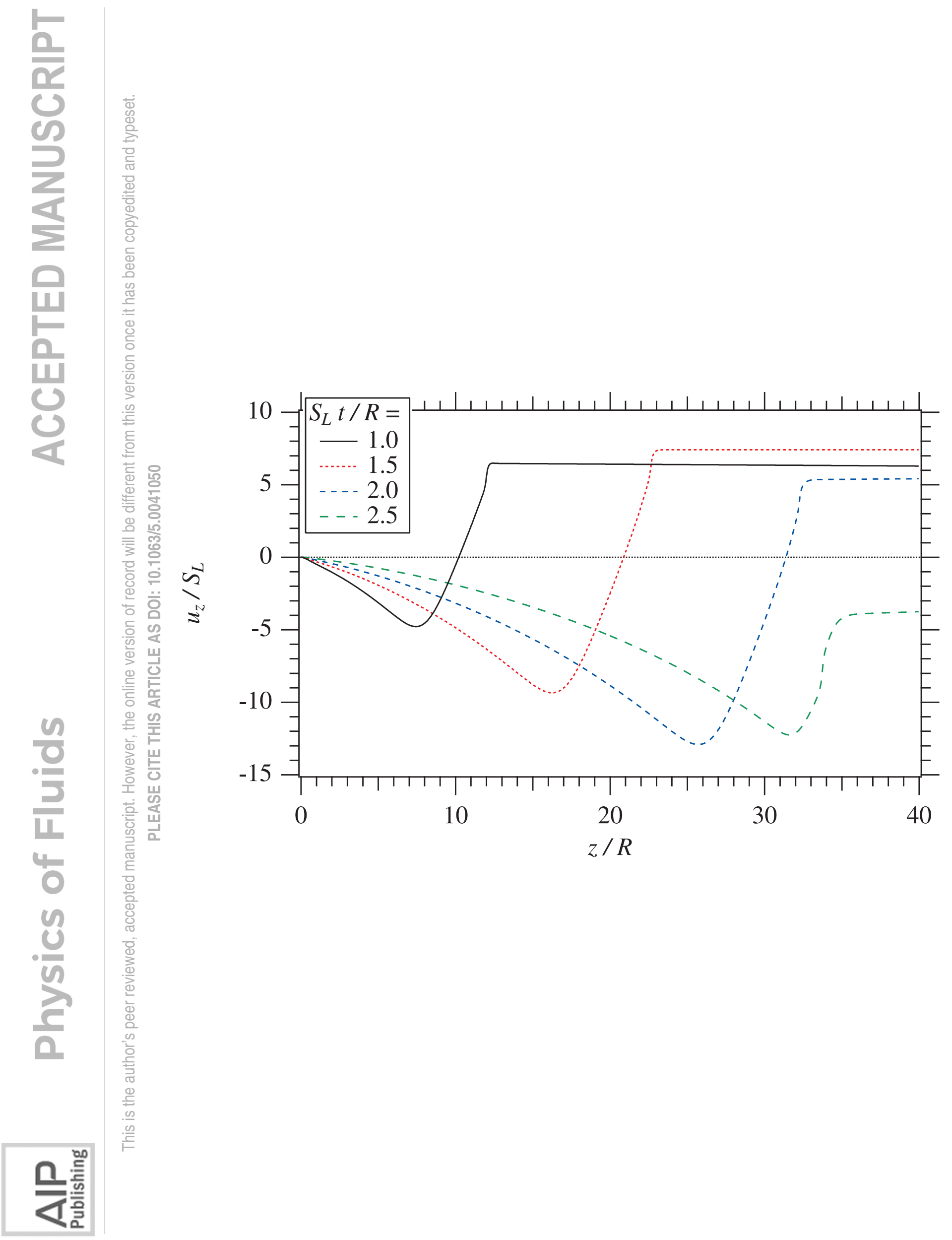




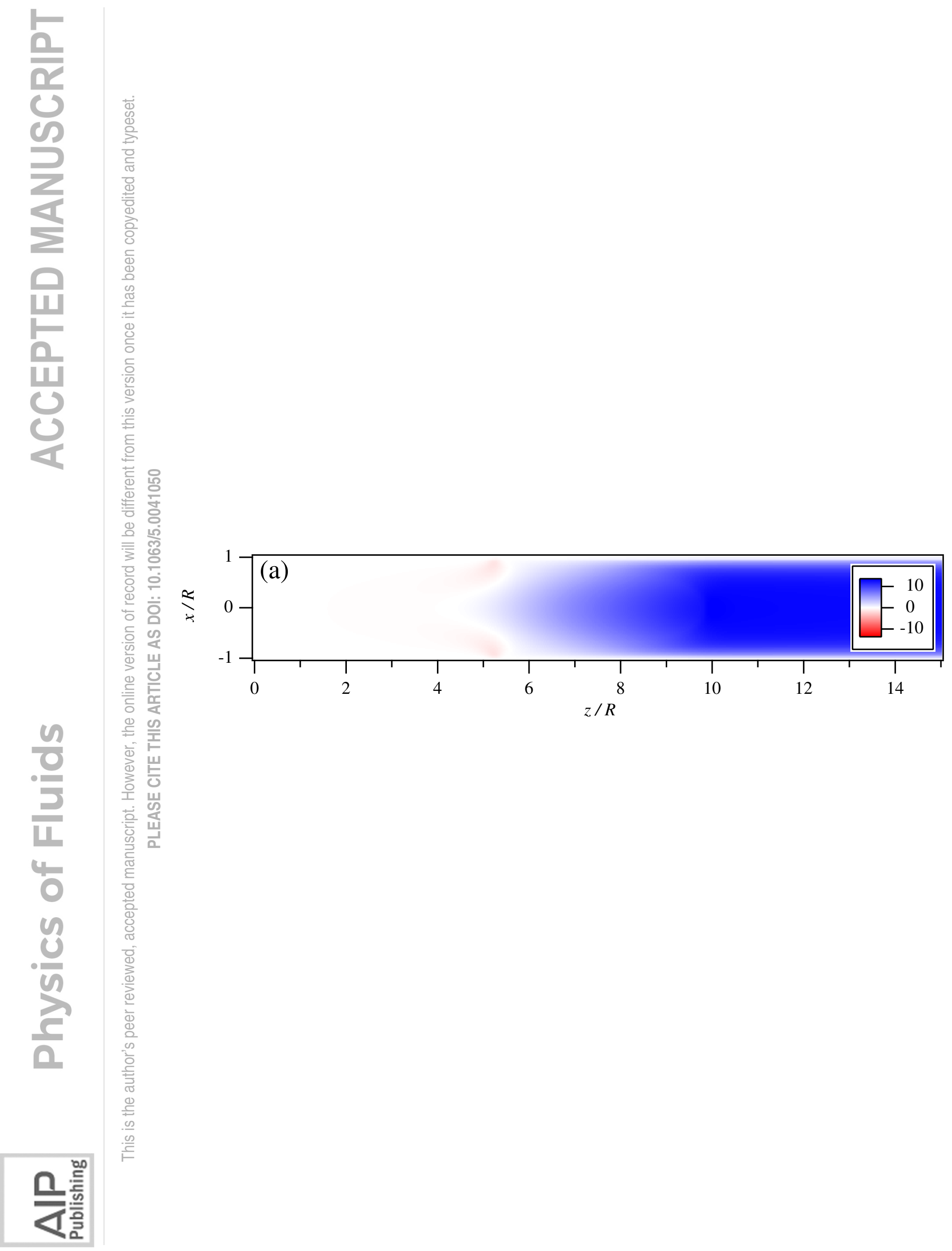




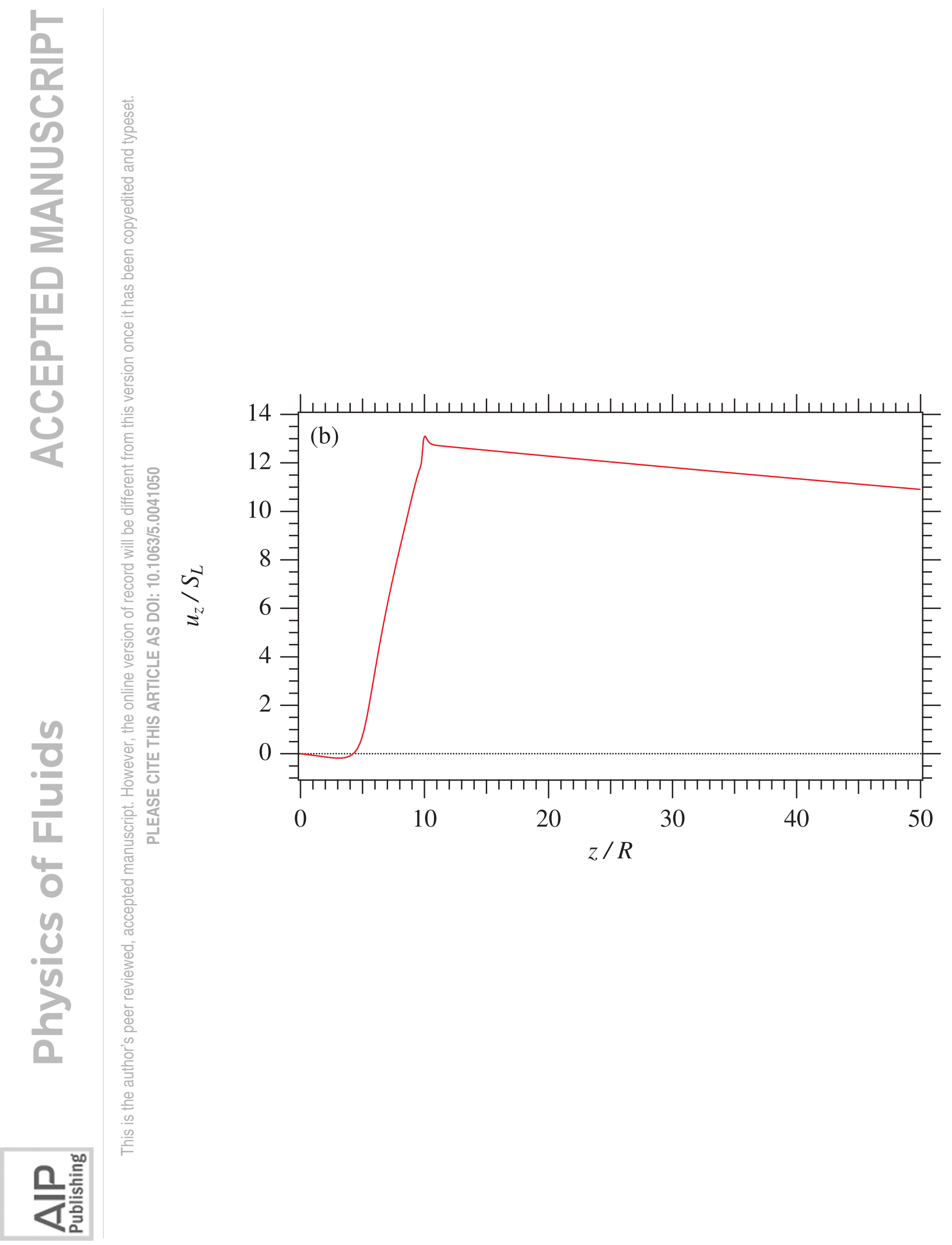




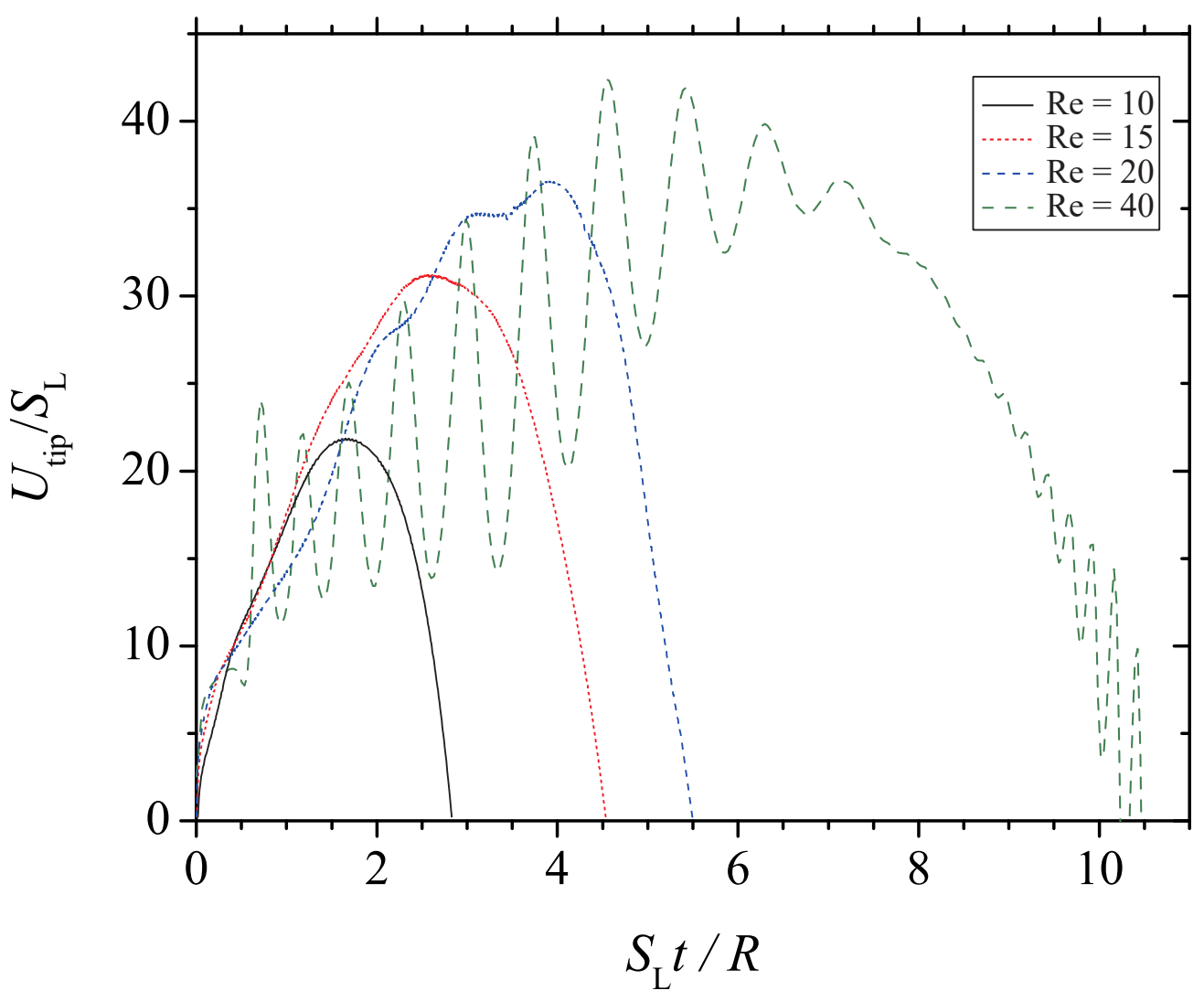




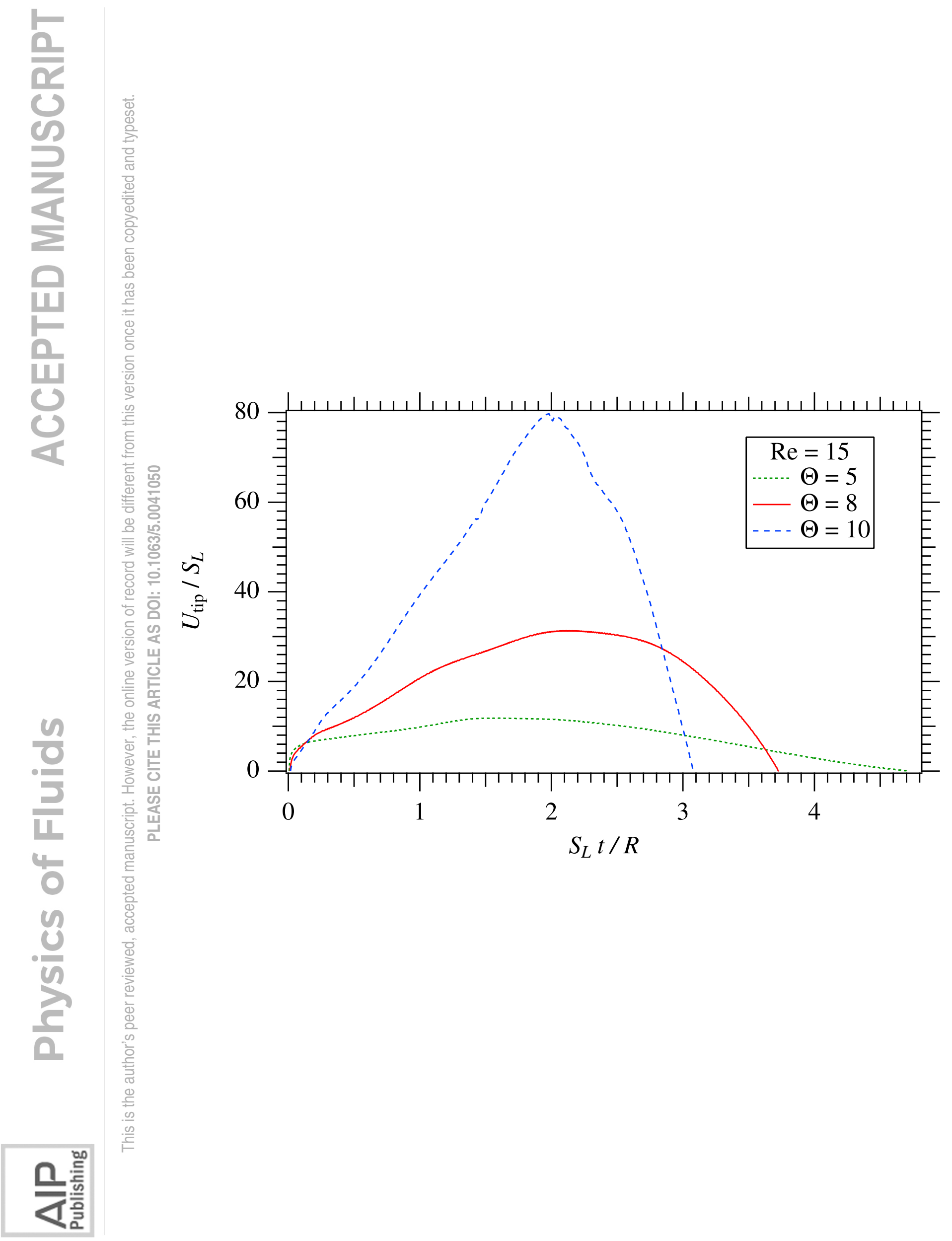




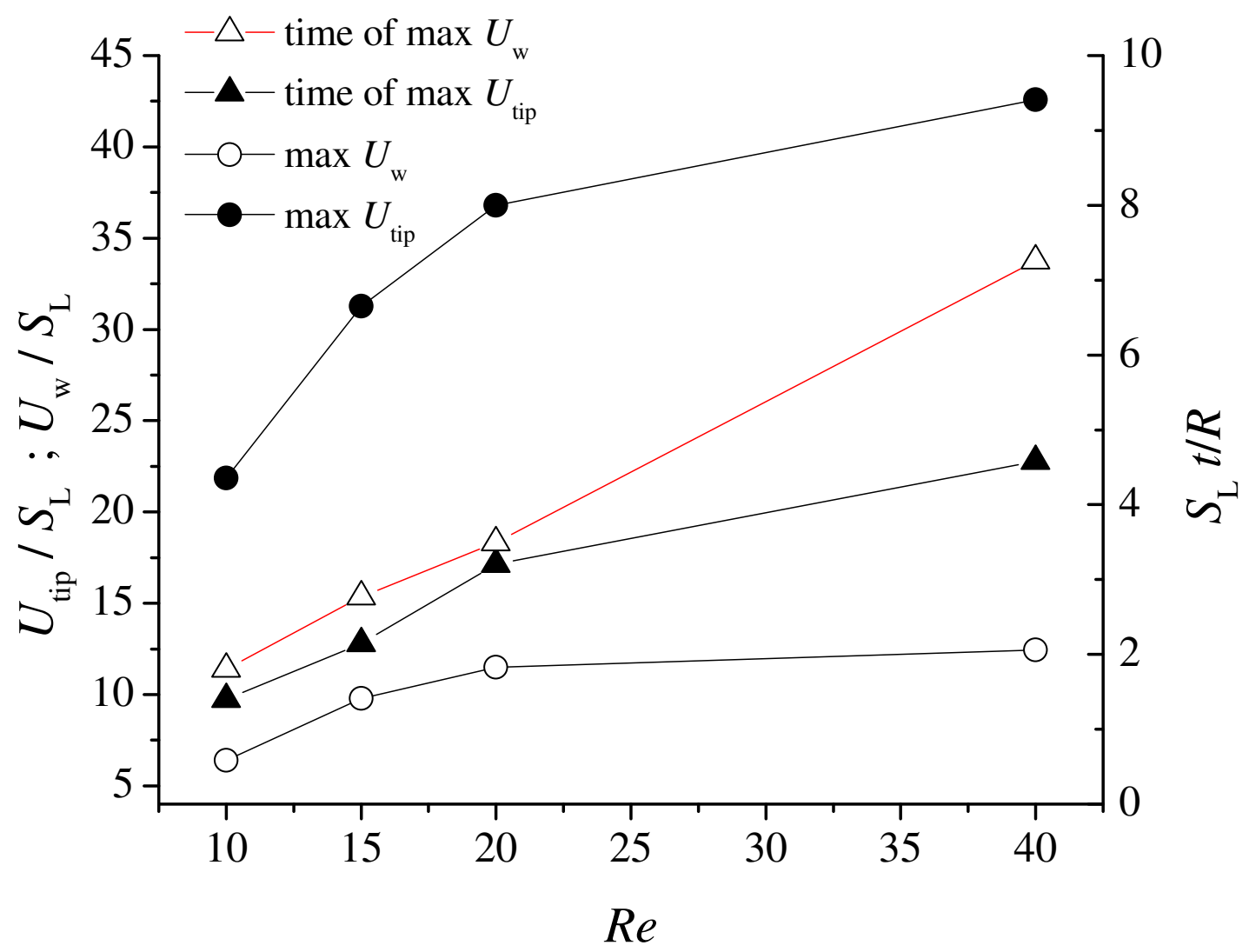




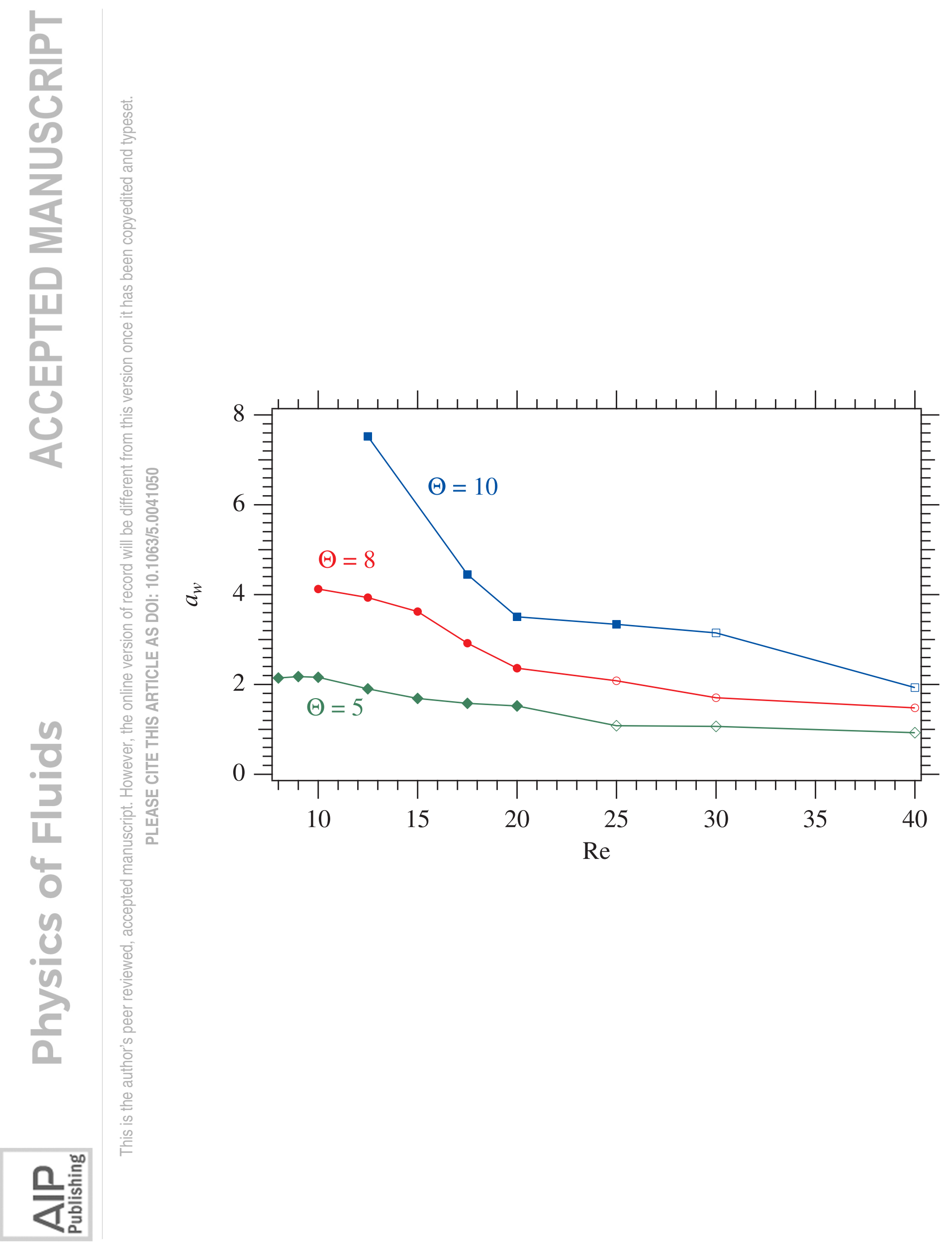




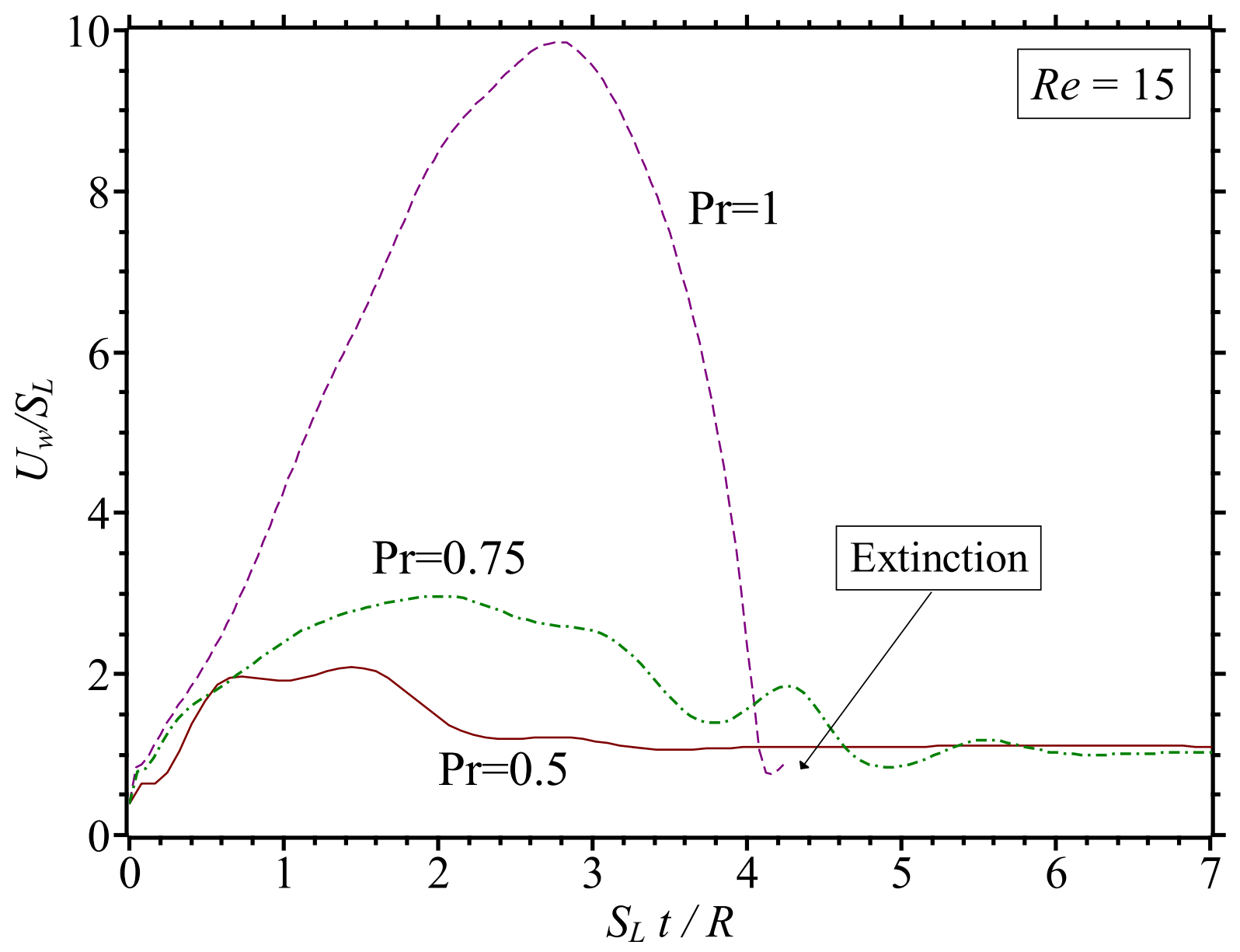




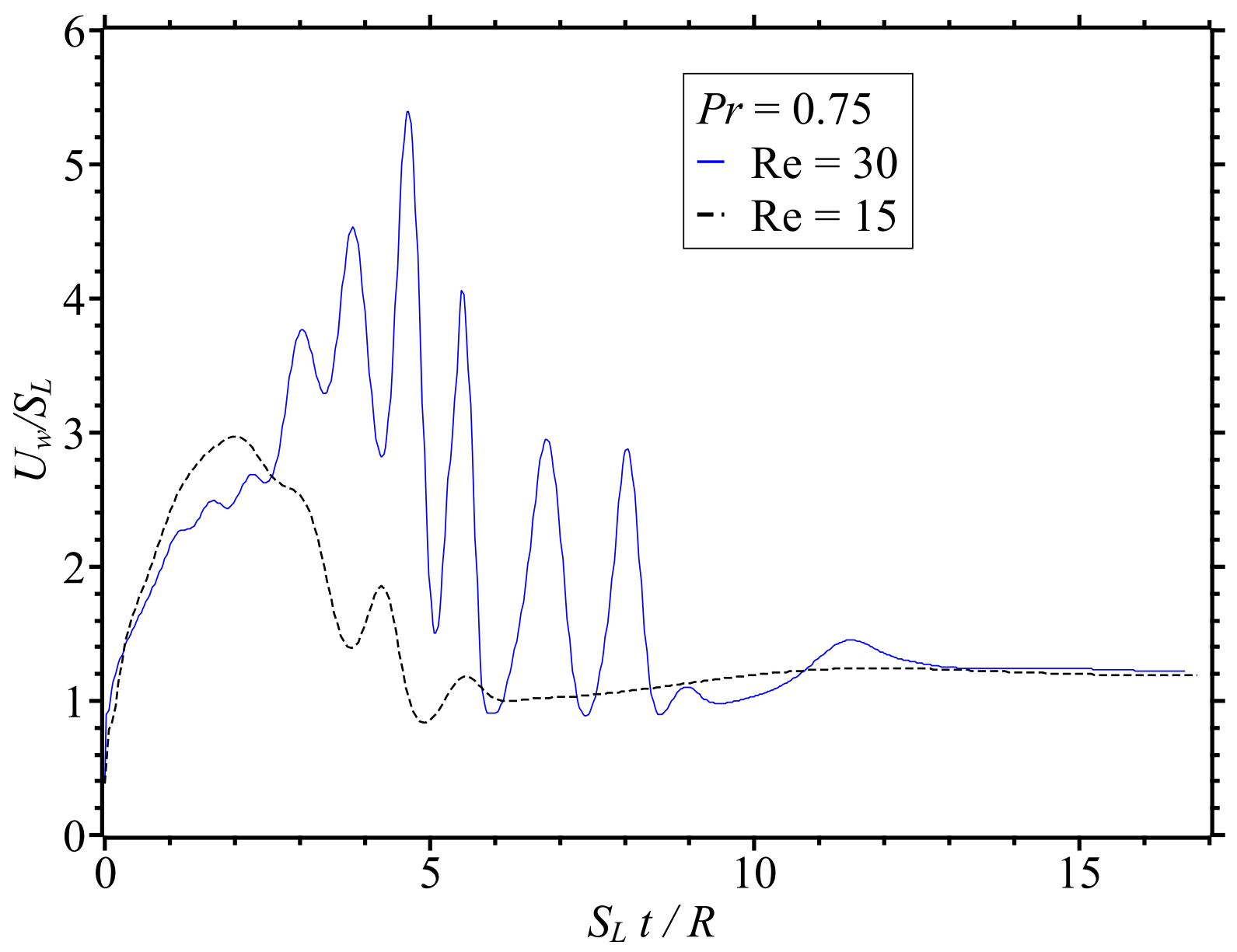

兄喜产 


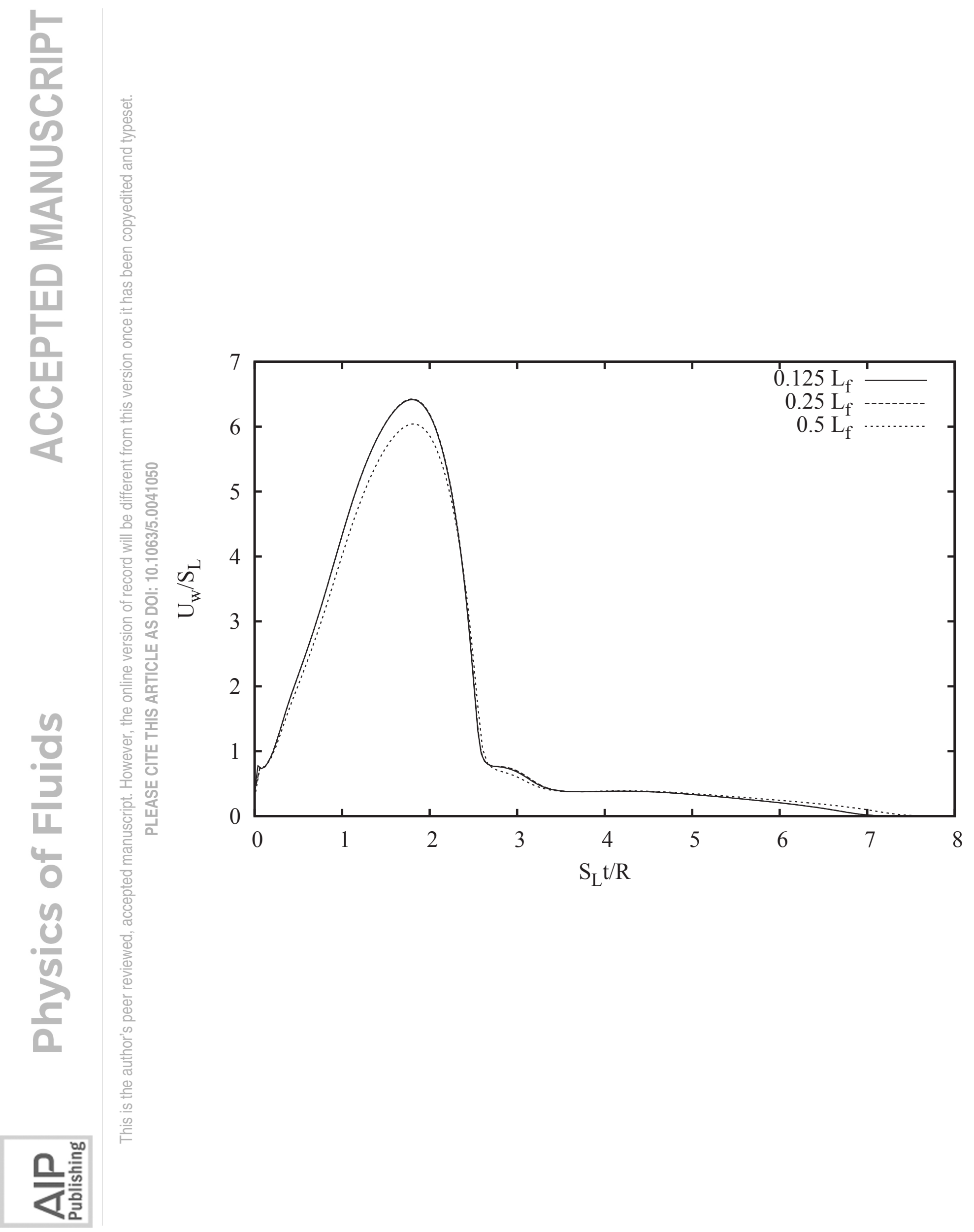

